\title{
FORCE AND TORQUE OF AN ELECTROMAGNETICALLY LEVITATED METAL SPHERE
}

\author{
BY \\ G. LOHÖFER
}

Institute for Space Simulation, German Aerospace Research Establishment, D-5000 Köln 90, Germany

\begin{abstract}
The Lorentz force and torque exerted on an electrically conducting sphere exposed to an external, time-varying magnetic field are analytically calculated. The external magnetic field is generated by a set of sinusoidally alternating, but otherwise arbitrary, current density fields of different frequencies and phases. Expressions for the force and torque in a laboratory frame of reference, which is more convenient for application, are also given. Finally, the special cases of rotational and mirror-symmetric external current density fields are treated in more detail.
\end{abstract}

1. Introduction. Eddy currents are induced when an electrically conducting sample is placed in an external, alternating magnetic field. These eddy currents give rise to two effects. On the one hand, they heat and melt the sample. On the other hand, the interaction of the eddy currents with the primary, nonuniform magnetic field results in a Lorentz force that can support the solid or liquid sample against gravity. This process, known as "electromagnetic levitation", is an important instrument in materials science, when either very hot metallic melts or metallic melts in the undercooled state have to be handled contactlessly. Moreover, this technique, especially when it is performed under microgravity conditions [1,2], establishes the basis for improved measuring methods of different thermophysical properties of undercooled metallic melts [3].

For a levitated specimen the questions of interest are: What is the power absorbed in the specimen, and what is the force and torque exerted on it by the external, alternating magnetic field. In answering these problems several points have to be considered. An investigation of the sample stability in the magnetic field supposes in general a three-dimensional treatment of the problem. Moreover, demands for high efficiency and independent control of positioning and heating of the sample require at least two magnetic fields of different frequencies or phases $[1,2,4]$. In regarding such points the present work goes beyond former publications $[5,6]$ that suppose cylindrical symmetry with a common axis of symmetry for the field and the spherical sample. The simplified 3-d theory of [7], and [8], on the other hand,

Received August 22, 1991.

1991 Mathematics Subject Classification. Primary 78A55, 42C10, 42C20.

(C)1993 Brown University 
which is also discussed in [9], is restricted to slightly inhomogeneous magnetic fields, a condition that is seldom satisfied in reality.

Here, we calculate basically the time-averaged force and torque exerted on an electrically conducting sphere in an external, alternating magnetic field. This magnetic field is supposed to be generated by a given set of sinusoidally alternating, but otherwise arbitrary, current density fields of different frequencies and phases. The present work is based on a paper [9] in which the induced eddy currents and the electrical power absorption of the sphere have already been calculated.

For a simple formulation of the boundary conditions at the surface of the sphere, we firstly calculate force and torque supposing a frame of reference whose origin is fixed at the center of the sphere. This assumption, which also restricts the results of [5], [6], and [9], has, however, the disadvantage that any movement of the sphere results in a coordinate transformation of the given external current density functions, which is inconvenient in application. To overcome this restriction, we also derive expressions for the force, the torque, and the power absorption from [9] that are based on a laboratory frame of reference fixed at any suitable point (e.g., point of symmetry) of the given external current density fields. Finally, these results are applied to the special cases of rotational, and mirror-symmetric external current density fields.

2. Foundations. In terms of the vector potential $\mathbf{A}(\mathbf{x}, t)$, which is uniquely defined [10] by the magnetic field

$$
\boldsymbol{\nabla} \times \mathbf{A}(\mathbf{x}, t)=\mathbf{B}(\mathbf{x}, t),
$$

the Coulomb gauge

$$
\boldsymbol{\nabla} \cdot \mathbf{A}(\mathbf{x}, t)=0,
$$

and the condition $\mathbf{A}(\mathbf{x}, t) \rightarrow \mathbf{0}$ for $|\mathbf{x}| \rightarrow \infty$, the basic Maxwell equations read in the present case [9] for all $\mathbf{x} \in \mathbb{R}^{3}$

$$
\begin{gathered}
\mathbf{j}(\mathbf{x}, t)=-\sigma(\mathbf{x}) \frac{\partial}{\partial t} \mathbf{A}(\mathbf{x}, t), \\
\mu_{0} \mathbf{j}(\mathbf{x}, t)=-\Delta \mathbf{A}(\mathbf{x}, t),
\end{gathered}
$$

where $\mathbf{j}(\mathbf{x}, t)$ is the current density field, $\sigma(\mathbf{x})$ the dc conductivity, and $\mu_{0}$ the magnetic induction constant.

In the following we identify our spherical sample with the closed point set $\bar{S}:=$ $\left\{\mathbf{x}:|\mathbf{x}| \leq R_{S}\right\}$ of radius $R_{S}$ and center in the origin of the coordinate system. (Throughout this paper all terms concerning this sphere are labeled by the index $S$.) In order to separate $\bar{S}$ from the given set of sinusoidally alternating, "external" current density fields

$$
\mathbf{j}_{E}(\mathbf{x}, t):= \begin{cases}\mathbf{0} & \text { for all } \mathbf{x} \in S^{+} \\ \mathbf{j}(\mathbf{x}, t) \equiv \sum_{n} \mathbf{j}_{n}(\mathbf{x}) \cos \left(\omega_{n} t+\alpha_{n}\right) & \text { for all } \mathbf{x} \in \mathbb{R}^{3} \backslash S^{+}\end{cases}
$$

we define a second concentric spherical point set: $S^{+}:=\left\{\mathbf{x}:|\mathbf{x}|<R_{S^{+}}, R_{S^{+}}>R_{S}\right\}$; see Fig. 1. Inside of the gap $S^{+} \mid \bar{S}$ we assume $\sigma(\mathbf{x})=0$ and obtain there $\mathbf{j}(\mathbf{x}, t)=\mathbf{0}$ from (3). Assuming integrability we see that the given external current density field 


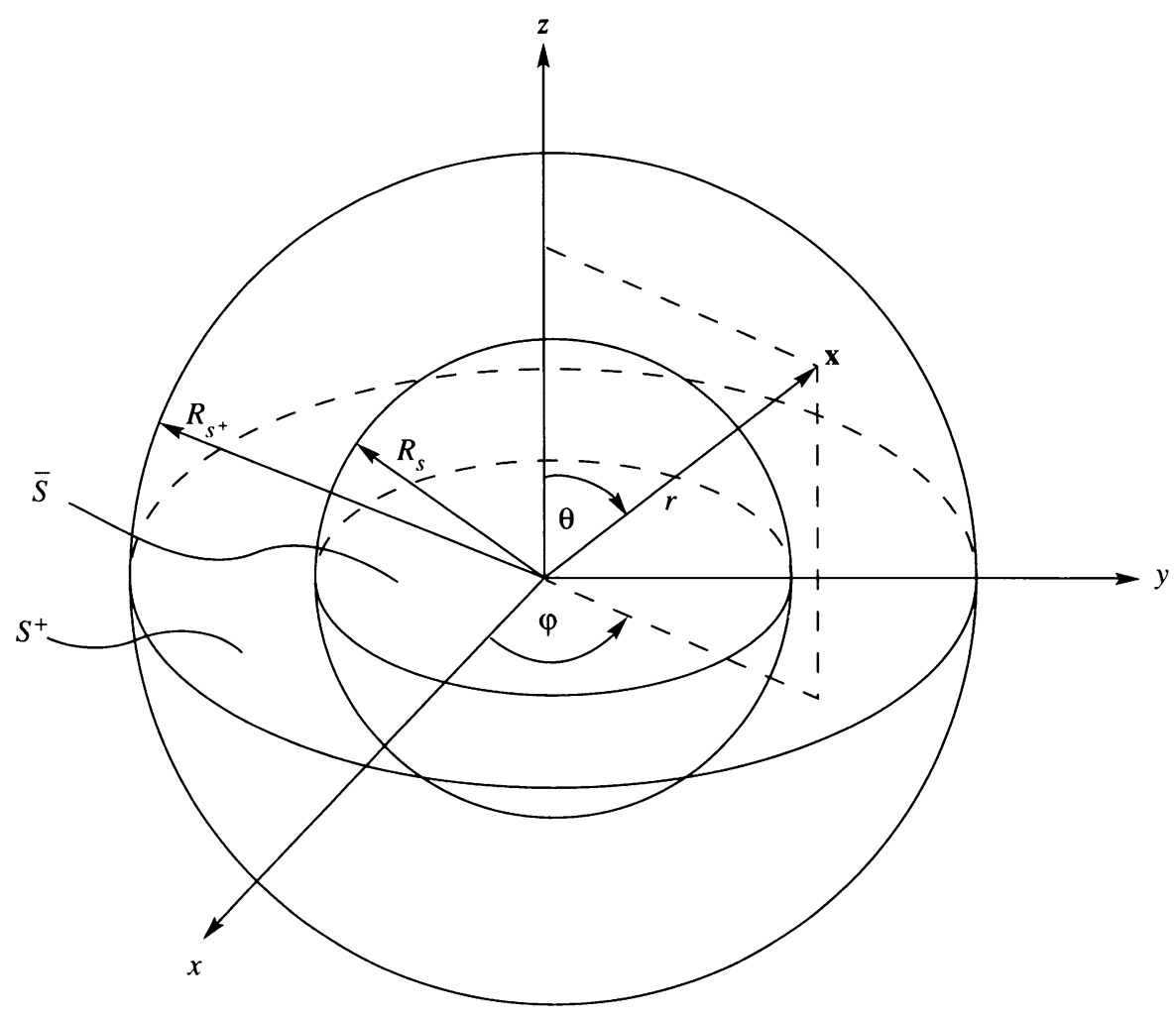

FIG. 1. Arrangement of the metal sphere $\bar{S}$ of radius $R_{S}$, the surrounding sphere $S^{+}$of radius $R_{S^{+}}$, and the applied frame of reference.

$\mathbf{j}_{\mathrm{E}}(\mathbf{x}, t)$ can be used to define the "external" vector potential $\mathbf{A}_{\mathbf{E}}(\mathbf{x}, t)$ by [10]:

$$
\mathbf{A}_{\mathrm{E}}(\mathbf{x}, t):=\frac{\mu_{0}}{4 \pi} \int_{\mathbb{R}^{3} \backslash S^{+}} \frac{\mathbf{j}_{\mathrm{E}}\left(\mathbf{x}^{\prime}, t\right)}{\left|\mathbf{x}-\mathbf{x}^{\prime}\right|} d^{3} x^{\prime}
$$

Finally, we define the eddy current density field inside the sphere by

$$
\mathbf{j}_{S}(\mathbf{x}, t):= \begin{cases}\mathbf{j}(\mathbf{x}, t) & \text { for all } \mathbf{x} \in \bar{S} \\ \mathbf{0} & \text { for all } \mathbf{x} \in \mathbb{R}^{3} \backslash \bar{S}\end{cases}
$$

Then, the assumption of a constant conductivity $\sigma_{S}$ inside $\bar{S}$ and the partition of the total vector potential $\mathbf{A}(\mathbf{x}, t)$ in

$$
\mathbf{A}(\mathbf{x}, t)=\mathbf{A}_{S}(\mathbf{x}, t)+\mathbf{A}_{\mathrm{E}}(\mathbf{x}, t)
$$

leads together with Eqs. (3)-(7) to

$$
\begin{array}{ll}
\mathbf{j}_{S}(\mathbf{x}, t)=-\sigma_{S} \frac{\partial}{\partial t} \mathbf{A}(\mathbf{x}, t) & \text { for all } \mathbf{x} \in \bar{S} \\
\Delta \mathbf{A}_{S}(\mathbf{x}, t)=-\mu_{0} \mathbf{j}_{S}(\mathbf{x}, t) & \text { for all } \mathbf{x} \in \bar{S}
\end{array}
$$


and

$$
\Delta \mathbf{A}_{S}(\mathbf{x}, t)=\mathbf{0} \text { for all } \mathbf{x} \in \mathbb{R}^{3} \backslash \bar{S} .
$$

Subject to the boundary conditions that $\mathbf{A}_{S}(\mathbf{x}, t)$ is continuously differentiable in $\mathbb{R}^{3}$ and $\mathbf{A}_{S}(\mathbf{x}, t) \rightarrow \mathbf{0}$ as $|\mathbf{x}| \rightarrow \infty$, the vector potential $\mathbf{A}_{S}(\mathbf{x}, t)$ and hence the current density $\mathbf{j}_{S}(\mathbf{x}, t)$ inside the sphere $\bar{S}$ can be uniquely determined from these differential equations.

In order to satisfy the boundary conditions at the surface of $\bar{S}$ it is convenient to represent the solution in spherical coordinates $\mathbf{x}=(r, u, \varphi)$, where $u:=\cos \theta$, with origin in the center of $\bar{S}$. When the exponentially decaying term that describes the time development of the initial state is neglected, the resulting stationary solution of the eddy current density $\mathbf{j}_{S}(\mathbf{x}, t)$ inside the sphere $\bar{S}$ generated by the given external current densities $\mathbf{j}_{n}(\mathbf{x})$ outside of $S^{+}$then reads [9]

$$
\begin{aligned}
\mathbf{j}_{S}^{\infty}(r, u, \varphi, t)= & \frac{2}{R_{S}^{3 / 2}} \sum_{n} \sum_{l=0}^{\infty} \sum_{m=-l}^{+l} \sum_{k=1}^{\infty} \frac{R_{S}^{l} \mathbf{I}_{n, l, m}}{J_{l+1 / 2}\left(x_{l+1 / 2, k}\right)} \frac{2 q_{n}^{2}}{\sqrt{4 q_{n}^{4}+x_{l+1 / 2, k}^{4}}} \\
& \times r^{-1 / 2} J_{l+1 / 2}\left(x_{l+1 / 2, k} \frac{r}{R_{S}}\right) Y_{l}^{m}(u, \varphi) \cos \left(\omega_{n} t+\alpha_{n}+\psi_{n, k, l}\right)
\end{aligned}
$$

with the complex, vectorial coefficients

$$
\begin{aligned}
\mathbf{I}_{n, l, m} & :=\int_{R_{S^{+}}}^{\infty} \int_{-1}^{+1} \int_{0}^{2 \pi} \mathbf{j}_{n}(r, u, \varphi) r^{-l+1} Y_{l}^{m^{*}}(u, \varphi) d \varphi d u d r \\
& \equiv \frac{1}{a_{l, m}} \int_{\mathbb{R}^{3} \backslash S^{+}} X_{l}^{m^{*}}(\mathbf{x}) \mathbf{j}_{n}(\mathbf{x}) d^{3} x,
\end{aligned}
$$

where the definitions of $a_{l, m}$ and the kernel $X_{l}^{m}$ are given in (87) and (88), respectively. Furthermore, $J_{l+1 / 2}$ denotes the half-integer order Bessel functions, $x_{l+1 / 2, k}$ the positive real zeros of $J_{l-1 / 2}$, and $Y_{l}^{m}$ the spherical harmonics (see Appendix D). The angle $\psi_{n, k, l}$, that specifies the phase shift between the external (5) and the induced current density (12) of mode $l, k$ is determined by

$$
\cos \psi_{n, k, l}=-\frac{2 q_{n}^{2}}{\sqrt{4 q_{n}^{4}+x_{l+1 / 2, k}^{4}}}, \quad \sin \psi_{n, k, l}=-\frac{x_{l+1 / 2, k}^{2}}{\sqrt{4 q_{n}^{4}+x_{l+1 / 2, k}^{4}}},
$$

and depends on the skin depth $\delta_{n}$ via

$$
q_{n}:=\frac{R_{S}}{\delta_{n}}, \quad \delta_{n}:=\sqrt{\frac{2}{\mu_{0} \sigma_{S} \omega_{n}}} .
$$

With the help of the series representation for $\mathbf{j}_{S}^{\infty}(\mathbf{x}, t)$, which is uniformly convergent throughout $\bar{S}$, the time-averaged power absorbed in the sphere can be written in the form [9]

$$
\bar{P}_{S}=\frac{1}{2 R_{S} \sigma_{S}} \sum_{n} \sum_{n^{\prime}} \sum_{l=0}^{\infty} \sum_{m=-l}^{+l} H_{l}\left(q_{n}\right) \delta_{\omega_{n}, \omega_{n^{\prime}}} \cos \left(\alpha_{n}-\alpha_{n^{\prime}}\right) R_{S}^{2 l} \mathbf{I}_{n^{\prime}, l, m} \cdot \mathbf{I}_{n, l, m}^{*},
$$


where

$$
H_{l}\left(q_{n}\right):=-\operatorname{Re}\left\{(1+i) q_{n} \frac{J_{l+1 / 2}\left((1+i) q_{n}\right)}{J_{l-1 / 2}\left((1+i) q_{n}\right)}\right\} .
$$

3. Time-averaged force. The time-averaged Lorentz force exerted by the magnetic field $\mathbf{B}(\mathbf{x}, t)$ on the sphere $\bar{S}$ is defined [10] by

$$
\begin{aligned}
\overline{\mathbf{F}}_{S} & :=\lim _{T \rightarrow \infty} \frac{1}{T} \int_{0}^{T} \int_{\bar{S}} \mathbf{j}(\mathbf{x}, t) \times[\nabla \times \mathbf{A}(\mathbf{x}, t)] d^{3} x d t \\
& =\lim _{T \rightarrow \infty} \frac{1}{T} \int_{0}^{T}\left(\int_{\bar{S}} \nabla\{\mathbf{A}(\mathbf{x}, t)\} \cdot \mathbf{j}_{S}(\mathbf{x}, t) d^{3} x-\int_{\bar{S}} \mathbf{j}(\mathbf{x}, t) \cdot \nabla \mathbf{A}(\mathbf{x}, t) d^{3} x\right) d t,
\end{aligned}
$$

where (1), (7), and the well-known triple vector product formula have been used. In ambiguous cases those vectorial or dyadic fields that are differentiated by the nabla operator are enclosed in braces. Equation (18) contains identically vanishing terms that will be identified in the following.

Using the Gaussian integral theorem, the second term of (18) can be transformed into

$$
\int_{\bar{S}} \mathbf{j}(\mathbf{x}, t) \cdot \boldsymbol{\nabla} \mathbf{A}(\mathbf{x}, t) d^{3} x=\oint_{\partial S} \mathbf{n}(\mathbf{x}) \cdot \mathbf{j}(\mathbf{x}, t) \mathbf{A}(\mathbf{x}, t) d \sigma-\int_{\bar{S}} \mathbf{A}(\mathbf{x}, t) \boldsymbol{\nabla} \cdot \mathbf{j}(\mathbf{x}, t) d^{3} x=\mathbf{0},
$$

where $\mathbf{n}(\mathbf{x})$ is the normal vector to the spherical surface $\partial S$. The integrands of both integrals vanish identically in their respective regions of integration. This is due to the fact that the Coulomb gauge (2) and Eq. (4) immediately result in

$$
\boldsymbol{\nabla} \cdot \mathbf{j}(\mathbf{x}, t)=0
$$

for all $\mathbf{x} \in \mathbb{R}^{3}$. Furthermore, due to Gauss's integral theorem, Eq. (20) again implies that the normal component of $\mathbf{j}(\mathbf{x}, t)$ is continuous throughout a smooth surface. Since, see Sec. $2, \mathbf{j}(\mathbf{x}, t)=\mathbf{0}$ inside a spherical shell around $\bar{S}$, we also find that

$$
\mathbf{n}(\mathbf{x}) \cdot \mathbf{j}(\mathbf{x}, t)=0 \text { for all } \mathbf{x} \in \partial S .
$$

For a further simplification of (18) we remark first that subject to the boundary condition given in Sec. 2

$$
\mathbf{A}_{S}(\mathbf{x}, t)=\frac{\mu_{0}}{4 \pi} \int_{\bar{S}} \frac{\mathbf{j}_{S}\left(\mathbf{x}^{\prime}, t\right)}{\left|\mathbf{x}-\mathbf{x}^{\prime}\right|} d^{3} x^{\prime}
$$

is the unique solution of (10) and (11) [11, Chap. IV, 1]. According to the partition of the vector potential (8) the first term on the right-hand side of (18) contains the integral

$$
\int_{\bar{S}} \boldsymbol{\nabla}\left\{\mathbf{A}_{S}(\mathbf{x}, t)\right\} \cdot \mathbf{j}_{S}(\mathbf{x}, t) d^{3} x=\frac{\mu_{0}}{4 \pi} \int_{\bar{S}} \int_{\bar{S}} \mathbf{j}_{S}(\mathbf{x}, t) \cdot \mathbf{j}_{S}\left(\mathbf{x}^{\prime}, t\right) \frac{\mathbf{x}-\mathbf{x}^{\prime}}{\left|\mathbf{x}-\mathbf{x}^{\prime}\right|^{3}} d^{3} x^{\prime} d^{3} x=\mathbf{0} .
$$

The interchange of the order of differentiation and integration is justified in [11, Chap. IV, 1]. The last expression on the right-hand side of (23) disappears, because 
an interchange of the order of integration changes the sign but keeps the double integral otherwise form-invariant. From this discussion we conclude that

$$
\overline{\mathbf{F}}_{S}=\lim _{T \rightarrow \infty} \frac{1}{T} \int_{0}^{T} \int_{\bar{S}} \nabla\left\{\mathbf{A}_{\mathrm{E}}(\mathbf{x}, t)\right\} \cdot \mathbf{j}_{S}^{\infty}(\mathbf{x}, t) d^{3} x d t .
$$

In (24) we consider only the stationary part: $\mathbf{j}_{S}^{\infty}(\mathbf{x}, t)$, i.e., (12), of the eddy current solution, assuming that the physically interesting observation time scale is long compared to the decay of the initial state of $\mathbf{j}_{S}(\mathbf{x}, t)$ (for an estimation see [9, Sec. 3]).

To calculate the volume integral in (24) explicitly, we first investigate the dyadic tensor $\boldsymbol{\nabla} \mathbf{A}_{\mathrm{E}}(\mathbf{x}, t)$ for $\mathbf{x} \in \bar{S}$ in more detail. Using (6) we find

$$
\begin{aligned}
\boldsymbol{\nabla} \mathbf{A}_{\mathbf{E}}(\mathbf{x}, t) & =\frac{\mu_{0}}{4 \pi} \int_{\mathbb{R}^{3} \backslash S^{+}} \nabla_{\mathbf{x}} \frac{\mathbf{j}_{\mathrm{E}}\left(\mathbf{x}^{\prime}, t\right)}{\left|\mathbf{x}-\mathbf{x}^{\prime}\right|} d^{3} x^{\prime} \\
& =-\frac{\mu_{0}}{4 \pi} \int_{\mathbb{R}^{3} \backslash S^{+}} \nabla_{\mathbf{x}^{\prime}}\left\{\frac{1}{\left|\mathbf{x}-\mathbf{x}^{\prime}\right|}\right\} \mathbf{j}_{\mathrm{E}}\left(\mathbf{x}^{\prime}, t\right) d^{3} x^{\prime} .
\end{aligned}
$$

Using for $\left|\mathbf{x}-\mathbf{x}^{\prime}\right|^{-1}$ with $\mathbf{x} \in \bar{S}$ and $\mathbf{x}^{\prime} \in \mathbb{R}^{3} \backslash S^{+}$the series representation (107), which (see Appendix C) may be differentiated and integrated termwise, we obtain using (5)

$$
\boldsymbol{\nabla} \mathbf{A}_{\mathbf{E}}(\mathbf{x}, t)=-\mu_{0} \sum_{n} \sum_{l=0}^{\infty} \sum_{m=-l}^{+l} \frac{r^{l} Y_{l}^{m^{*}}(u, \varphi)}{2 l+1} \mathrm{~F}_{n, l, m}^{*} \cos \left(\omega_{n} t+\alpha_{n}\right)
$$

with the dyadic tensor

$$
\begin{aligned}
\mathbf{F}_{n, l, m}^{*} & :=\int_{\mathbb{R}^{3} \backslash S^{+}} \nabla_{\mathbf{x}^{\prime}}\left\{r^{\prime-l-1} Y_{l}^{m}\left(u^{\prime}, \varphi^{\prime}\right)\right\} \mathbf{j}_{n}\left(\mathbf{x}^{\prime}\right) d^{3} x^{\prime} \\
& \equiv \frac{1}{a_{l, m}} \int_{\mathbb{R}^{3} \backslash S^{+}} \nabla_{\mathbf{x}^{\prime}}\left\{X_{l}^{m}\left(\mathbf{x}^{\prime}\right)\right\} \mathbf{j}_{n}\left(\mathbf{x}^{\prime}\right) d^{3} x^{\prime}
\end{aligned}
$$

in which the definitions of $X_{l}^{m}(\mathbf{x})$ and $a_{l, m}$ from (87) and (88) have been used. Contrary to the known integral coefficients $\mathbf{I}_{n, l, m}$ of (13), which occur in the eddy current solution $\mathbf{j}_{S}^{\infty}(\mathbf{x}, t)$ of (12), this integral contains the gradient of the kernel $X_{l}^{m}$. According to the results (86), (94)-(96) of Appendix B, $\mathrm{F}_{n, l, m}$ and $\mathbf{I}_{n, l^{\prime}, m^{\prime}}$ are, however, closely related, which leads to

$$
\mathbf{F}_{n, l, m}^{*}=\frac{\mathbf{e}_{\eta}^{*}}{\sqrt{2}} \frac{a_{l+1, m+1}}{a_{l, m}} \mathbf{I}_{n, l+1, m+1}^{*}-\frac{\mathbf{e}_{\eta}}{\sqrt{2}} \frac{a_{l+1, m-1}}{a_{l, m}} \mathbf{I}_{n, l+1, m-1}^{*}+\mathbf{e}_{z} \frac{a_{l+1, m}}{a_{l, m}} \mathbf{I}_{n, l+1, m}^{*} .
$$

The properties of the complex unit vector $\mathbf{e}_{\eta}:=\left(\mathbf{e}_{x}+i \mathbf{e}_{y}\right) / \sqrt{2}$ are given in Appendix B.

Provided in (26) and consequently also in (27) and (28) the same frame of reference is used as in (12) (see Fig. 1), the two series representations render possible an easy evaluation of the volume integral of (24). The uniform convergence of both series allows a term-by-term integration that can immediately be evaluated taking into account the orthonormality relation of spherical harmonics (104), the well-known 
integral and recurrence properties of the Bessel functions [12], and the properties of the numbers $x_{l+1 / 2, k}$, see Sec. 2 . Thus, we obtain for the time-averaged force

$$
\overline{\mathbf{F}}_{S}=2 \mu_{0} \sum_{n} \sum_{n^{\prime}} \sum_{l=0}^{\infty} \sum_{m=-l}^{+l} R_{S}^{2 l+1} \mathrm{~F}_{n^{\prime}, l, m}^{*} \cdot \mathbf{I}_{n, l, m} Q_{n, n^{\prime}, l},
$$

where $Q_{n, n^{\prime}, l}$ contains the time-averaging integral

$$
\begin{aligned}
Q_{n, n^{\prime}, l} & :=-2 q_{n}^{2} \sum_{k=1}^{\infty} \frac{\lim _{T \rightarrow \infty} \frac{1}{T} \int_{0}^{T} \cos \left(\omega_{n} t+\alpha_{n}+\psi_{n, k, l}\right) \cos \left(\omega_{n^{\prime}} t+\alpha_{n^{\prime}}\right) d t}{x_{l+1 / 2, k}^{2} \sqrt{4 q_{n}^{4}+x_{l+1 / 2, k}^{4}}} \\
& =-q_{n}^{2} \sum_{k=1}^{\infty} \frac{\lim _{T \rightarrow \infty}\left\{\delta_{\omega_{n}, \omega_{n^{\prime}}}\left[\cos \left(\alpha_{n}-\alpha_{n^{\prime}}+\psi_{n, k, l}\right)+R_{\omega_{n}}(T)\right]+\left(1-\delta_{\omega_{n}, \omega_{n^{\prime}}}\right) S_{\omega_{n}, \omega_{n^{\prime}}}(T)\right\}}{x_{l+1 / 2, k}^{2} \sqrt{4 q_{n}^{4}+x_{l+1 / 2, k}^{4}}} \\
& =\delta_{\omega_{n}, \omega_{n^{\prime}}} \sum_{k=1}^{\infty} \frac{2 q_{n}^{4} \cos \left(\alpha_{n}-\alpha_{n^{\prime}}\right)-q_{n}^{2} x_{l+1 / 2, k}^{2} \sin \left(\alpha_{n}-\alpha_{n^{\prime}}\right)}{x_{l+1 / 2, k}^{2}\left(4 q_{n}^{4}+x_{l+1 / 2, k}^{4}\right)}
\end{aligned}
$$

In the last sum the expressions for the circular functions of $\psi_{n, k, l}$ given in (14) have been inserted. The above calculations take into account that $R_{\omega_{n}}(T)$ and $S_{\omega_{n}, \omega_{n^{\prime}}}(T)$ can be estimated for $\omega_{n} \neq \omega_{n^{\prime}}$ in the following way:

$$
\left|R_{\omega_{n}}(T)\right| \leq \frac{1}{\omega_{n} T}, \quad\left|S_{\omega_{n}, \omega_{n^{\prime}}}(T)\right| \leq \frac{4}{\left|\omega_{n}-\omega_{n^{\prime}}\right| T} .
$$

Since property (91) of $X_{l}^{m}$ is analogously satisfied by $\mathbf{I}_{n, l, m}$, and utilizing (90) and (84), we finally get $\overline{\mathbf{F}}_{S}$ in a very compact form:

$$
\begin{aligned}
\overline{\mathbf{F}}_{S}= & \frac{\mu_{0}}{2} \sum_{n} \sum_{n^{\prime}} \sum_{l=0}^{\infty} \sum_{m=-l}^{+l} \delta_{\omega_{n}, \omega_{n^{\prime}}} \operatorname{Re}\left\{G_{l}\left(q_{n}\right) e^{-i\left(\alpha_{n}-\alpha_{n^{\prime}}\right)}\right\} R_{S}^{2 l+1} \\
& \times\left(\frac{a_{l+1, m+1}}{a_{l, m}} \operatorname{Re}\left\{\mathbf{I}_{n^{\prime}, l+1, m+1} \cdot \mathbf{I}_{n, l, m}^{*}\left(\mathbf{e}_{x}+i \mathbf{e}_{y}\right)\right\}+\frac{a_{l+1, m}}{a_{l, m}} \mathbf{I}_{n^{\prime}, l+1, m} \cdot \mathbf{I}_{n, l, m}^{*} \mathbf{e}_{z}\right),
\end{aligned}
$$

where

$$
\begin{aligned}
G_{l}\left(q_{n}\right) & :=\sum_{k=1}^{\infty} \frac{8 q_{n}^{4}-i 4 q_{n}^{2} x_{l+1 / 2, k}^{2}}{\left(4 q_{n}^{4}+x_{l+1 / 2, k}^{4}\right) x_{l+1 / 2, k}^{2}} \\
& =-\frac{J_{l+3 / 2}\left((1+i) q_{n}\right)}{(2 l+1) J_{l-1 / 2}\left((1+i) q_{n}\right)},
\end{aligned}
$$

and, see Appendix B,

$$
a_{l, m}:=(-1)^{l+m} \sqrt{\frac{4 \pi}{2 l+1}(l+m) !(l-m) !} .
$$

The equality of the two expressions in (33) is proved in Appendix A. 
Together with $\mathbf{I}_{n, l, m}$ from (13), Eqs. (32) and (33) describe the time-averaged Lorentz force on the sphere $\bar{S}$, generated by a set of given, sinusoidally alternating current density fields of the form: $\mathbf{j}_{n}(\mathbf{x}) \cos \left(\omega_{n} t+\alpha_{n}\right)$ in an area outside of $S^{+}$(cf. Sec. 2). In principle, (32) is time independent. According to (31), Eq. (32) represents, however, a good approximation also in the case of time-dependent parameters provided their time scale $T$ satisfies $T \gg \omega_{n}^{-1}$ and $T \gg\left|\omega_{n}-\omega_{n^{\prime}}\right|^{-1}$, and provided $T$ is long compared to the decay time of the initial state of $\mathbf{j}_{S}(\mathbf{x}, t)$ (for an estimation see $\left[9\right.$, Sec. 3]). The occurrence of the Kronecker symbol $\delta_{\omega_{n}, \omega_{n^{\prime}}}$ in this case implies that Lorentz forces resulting from external current density distributions of different frequencies are independent of each other, which means that they can simply be added. This is, however, different for current density fields of equal frequencies but different phases.

Equation (13) shows that, roughly speaking, (32) essentially represents an expansion of $\overline{\mathbf{F}}_{S}$ in powers of $R_{S} / r$, where $r$ denotes the distances of the external current elements from the center of the sphere. Since $R_{S} / r<1$, this series converges absolutely for integrable $\mathbf{j}_{n}(\mathbf{x})$ (cf. Appendix C). The frequency dependence of every mode in this expansion is via $q_{n}$ and the skin depth $\delta_{n}$, see (15), given by $G_{l}\left(q_{n}\right)$. In practical applications the quantity $R_{S} / r$ may sometimes be small enough to consider only the first, or the first two nonvanishing terms in the series representation. For $l=1$ Eq. (32) agrees essentially with the theories of [5] and [6] (cf. [9, Sec. 1]).

Note that the functions $\mathbf{j}_{n}(\mathbf{x})$ as well as $\mathbf{I}_{n, l, m}$, the unit vectors $\mathbf{e}_{x}, \mathbf{e}_{y}, \mathbf{e}_{z}$, and hence also $\overline{\mathbf{F}}_{S}$ are defined in a frame of reference, the origin of which is fixed at the center of the sphere $\bar{S}$ (see, e.g., Fig. 1). This special choice of the coordinates makes possible a simple formulation of the boundary conditions in [9, Sec. 3]. It implies, however, that the functions $\mathbf{j}_{n}(\mathbf{x})$ change, if the relative position between the sphere (or the frame of reference) and the external current distributions changes. This problem is discussed in Sec. 5.

4. Time-averaged torque. The time-averaged torque on the sphere $\bar{S}$ around its center generated by the magnetic field $\mathbf{B}(\mathbf{x}, t)$ is defined [10] by

$$
\begin{aligned}
& \overline{\mathbf{N}}_{S}:= \lim _{T \rightarrow \infty} \frac{1}{T} \int_{0}^{T} \int_{\bar{S}} \mathbf{x} \times(\mathbf{j}(\mathbf{x}, t) \times[\nabla \times \mathbf{A}(\mathbf{x}, t)]) d^{3} x d t \\
&=\lim _{T \rightarrow \infty} \frac{1}{T} \int_{0}^{T}\left(\int_{\bar{S}} \mathbf{x} \times \nabla\{\mathbf{A}(\mathbf{x}, t)\} \cdot \mathbf{j}_{S}(\mathbf{x}, t) d^{3} x\right. \\
&\left.+\int_{\bar{S}} \mathbf{j}(\mathbf{x}, t) \cdot \nabla\{\mathbf{A}(\mathbf{x}, t)\} \times \mathbf{x} d^{3} x\right) d t
\end{aligned}
$$

The time-averaged torque around any other point is then related to $\overline{\mathbf{N}}_{S}$ and $\overline{\mathbf{F}}_{S}$ by Steiner's theorem. Since the following calculations for $\overline{\mathbf{N}}_{S}$ run similar to that for $\overline{\mathbf{F}}_{S}$, we will no longer display all steps in detail. Here too, Eq. (35) contains identically vanishing terms that will now be eliminated. 
By analogy with (19), the second term of (35) can be transformed into

$$
\begin{aligned}
\int_{\bar{S}} \mathbf{j}(\mathbf{x}, t) \cdot \boldsymbol{\nabla}\{\mathbf{A}(\mathbf{x}, t)\} \times \mathbf{x} d^{3} x \\
=\int_{\bar{S}} \boldsymbol{\nabla} \cdot\{\mathbf{j}(\mathbf{x}, t) \mathbf{A}(\mathbf{x}, t) \times \mathbf{x}\} d^{3} x+\int_{\bar{S}} \boldsymbol{\nabla} \cdot\{\mathbf{j}(\mathbf{x}, t) \mathbf{x}\} \times \mathbf{A}(\mathbf{x}, t) d^{3} x \\
=\oint_{\partial S} \mathbf{n}(\mathbf{x}) \cdot \mathbf{j}(\mathbf{x}, t) \mathbf{A}(\mathbf{x}, t) \times \mathbf{x} d \sigma+\int_{\bar{S}} \boldsymbol{\nabla} \cdot\{\mathbf{j}(\mathbf{x}, t)\} \mathbf{x} \times \mathbf{A}(\mathbf{x}, t) d^{3} x \\
\quad+\int_{\bar{S}} \mathbf{j}_{S}(\mathbf{x}, t) \cdot \nabla\{\mathbf{x}\} \times \mathbf{A}(\mathbf{x}, t) d^{3} x \\
=\int_{\bar{S}} \mathbf{j}_{S}(\mathbf{x}, t) \times \mathbf{A}(\mathbf{x}, t) d^{3} x \\
=\int_{\bar{S}} \mathbf{j}_{S}(\mathbf{x}, t) \times \mathbf{A}_{\mathrm{E}}(\mathbf{x}, t) d^{3} x .
\end{aligned}
$$

The first and second integral behind the second equality sign disappear because of (21) and (20). The final result profits again from the fact that with the help of (22)

$$
\int_{\bar{S}} \mathbf{j}_{S}(\mathbf{x}, t) \times \mathbf{A}_{S}(\mathbf{x}, t) d^{3} x=\frac{\mu_{0}}{4 \pi} \int_{\bar{S}} \int_{\bar{S}} \frac{\mathbf{j}_{S}(\mathbf{x}, t) \times \mathbf{j}_{S}\left(\mathbf{x}^{\prime}, t\right)}{\left|\mathbf{x}-\mathbf{x}^{\prime}\right|} d^{3} x^{\prime} d^{3} x=\mathbf{0} .
$$

Hence, according to (8), only the external vector potential $\mathbf{A}_{\mathbf{E}}(\mathbf{x}, t)$ is involved in (36).

This is also true for the first term on the right-hand side of (35), because, similar to (23), it contains the integral

$$
\begin{aligned}
\int_{\bar{S}} \mathbf{x} & \times \nabla\left\{\mathbf{A}_{S}(\mathbf{x}, t)\right\} \cdot \mathbf{j}_{S}(\mathbf{x}, t) d^{3} x \\
& =-\frac{\mu_{0}}{4 \pi} \int_{\bar{S}} \int_{\bar{S}} \mathbf{j}_{S}(\mathbf{x}, t) \cdot \mathbf{j}_{S}\left(\mathbf{x}^{\prime}, t\right) \frac{\mathbf{x} \times \mathbf{x}^{\prime}}{\left|\mathbf{x}-\mathbf{x}^{\prime}\right|^{3}} d^{3} x^{\prime} d^{3} x=\mathbf{0},
\end{aligned}
$$

which vanishes for the same reason that (23) vanishes. Consequently, we find that

$$
\begin{aligned}
\overline{\mathbf{N}}_{S}=\lim _{T \rightarrow \infty} \frac{1}{T} \int_{0}^{T}\left(\int_{\bar{S}} \mathbf{x}\right. & \times \nabla\left\{\mathbf{A}_{\mathrm{E}}(\mathbf{x}, t)\right\} \cdot \mathbf{j}_{S}^{\infty}(\mathbf{x}, t) d^{3} x \\
& \left.-\int_{\bar{S}} \mathbf{A}_{\mathrm{E}}(\mathbf{x}, t) \times \mathbf{j}_{S}^{\infty}(\mathbf{x}, t) d^{3} x\right) d t .
\end{aligned}
$$

Here too, we consider only the stationary part: $\mathbf{j}_{S}^{\infty}(\mathbf{x}, t)$, i.e., (12), of the eddy current solution.

Obviously, the two volume integrals in (39) are very similar to that in (24). Comparing (6) as well as

$$
\begin{aligned}
\mathbf{x} \times \boldsymbol{\nabla} \mathbf{A}_{\mathrm{E}}(\mathbf{x}, t) & =\frac{\mu_{0}}{4 \pi} \int_{\mathbf{R}^{3} \backslash S^{+}} \mathbf{x} \times \nabla_{\mathbf{x}} \frac{\mathbf{j}_{\mathbf{E}}\left(\mathbf{x}^{\prime}, t\right)}{\left|\mathbf{x}-\mathbf{x}^{\prime}\right|} d^{3} x^{\prime} \\
& =-\frac{\mu_{0}}{4 \pi} \int_{\mathbf{R}^{3} \backslash S^{+}} \mathbf{x}^{\prime} \times \nabla_{\mathbf{x}^{\prime}}\left\{\frac{1}{\left|\mathbf{x}-\mathbf{x}^{\prime}\right|}\right\} \mathbf{j}_{\mathrm{E}}\left(\mathbf{x}^{\prime}, t\right) d^{3} x^{\prime}
\end{aligned}
$$


with (25), we see that the time-averaged torque can, in complete analogy to (29), be expressed as

$$
\overline{\mathbf{N}}_{S}=2 \mu_{0} \sum_{n} \sum_{n^{\prime}} \sum_{l=0}^{\infty} \sum_{m=-l}^{+l} R_{S}^{2 l+1}\left(\mathbf{N}_{n^{\prime}, l, m}^{*} \cdot \mathbf{I}_{n, l, m}-\mathbf{M}_{n^{\prime}, l, m}^{*} \times \mathbf{I}_{n, l, m}\right) Q_{n, n^{\prime}, l},
$$

where the tensor $\mathrm{N}_{n, l, m}^{*}$ is obtained from $\mathrm{F}_{n, l, m}^{*}$ (27) by replacing the operator $\nabla_{\mathbf{x}^{\prime}}$ under the integral by $\mathbf{x}^{\prime} \times \nabla_{\mathbf{x}^{\prime}}$ :

$$
\mathrm{N}_{n, l, m}^{*}:=\frac{1}{a_{l, m}} \int_{\mathbb{R}^{3} \backslash S^{+}} \mathbf{x}^{\prime} \times \nabla_{\mathbf{x}^{\prime}}\left\{X_{l}^{m}\left(\mathbf{x}^{\prime}\right)\right\} \mathbf{j}_{n}\left(\mathbf{x}^{\prime}\right) d^{3} x^{\prime} .
$$

Analogously, the vector $\mathbf{M}_{n, l, m}^{*}$ is obtained by replacing formally $\nabla_{\mathbf{x}^{\prime}}$ by -1 :

$$
\mathbf{M}_{n, l, m}^{*}:=-\frac{1}{a_{l, m}} \int_{\mathbb{R}^{3} \backslash S^{+}} X_{l}^{m}\left(\mathbf{x}^{\prime}\right) \mathbf{j}_{n}\left(\mathbf{x}^{\prime}\right) d^{3} x^{\prime}=-\mathbf{I}_{n, l, m}^{*} .
$$

The quantity $Q_{n, n^{\prime}, l}$ is defined in (30). According to Appendix B, $\mathrm{N}_{n, l, m}$ can immediately be related to the expansion coefficients $\mathbf{I}_{n, l, m^{\prime}}$ defined in (13). With (97), (98) we obtain

$$
\begin{aligned}
\mathrm{N}_{n, l, m}^{*}= & -\frac{i \mathbf{e}_{\eta}^{*}}{\sqrt{2}} \frac{a_{l, m+1}}{a_{l, m}}(l-m) \mathbf{I}_{n, l, m+1}^{*}-\frac{i \mathbf{e}_{\eta}}{\sqrt{2}} \frac{a_{l, m-1}}{a_{l, m}}(l+m) \mathbf{I}_{n, l, m-1}^{*} \\
& +i \mathbf{e}_{z} m \mathbf{I}_{n, l, m}^{*} .
\end{aligned}
$$

In the same way and under the same conditions as in the derivation of $\overline{\mathbf{F}}_{S}$ in (32), we also obtain $\overline{\mathbf{N}}_{S}$ from the above equations in the compact form

$$
\begin{aligned}
\overline{\mathbf{N}}_{S}= & \frac{\mu_{0}}{2} \sum_{n} \sum_{n^{\prime}} \sum_{l=0}^{\infty} \sum_{m=-l}^{+l} \delta_{\omega_{n}, \omega_{n^{\prime}}} \operatorname{Re}\left\{G_{l}\left(q_{n}\right) e^{-i\left(\alpha_{n}-\alpha_{n^{\prime}}\right)}\right\} R_{S}^{2 l+1} \\
& \times\left(\mathbf{I}_{n^{\prime}, l, m} \times \mathbf{I}_{n, l, m}^{*}-\frac{a_{l, m+1}}{a_{l, m}}(l-m) \operatorname{Im}\left\{\mathbf{I}_{n^{\prime}, l, m+1} \cdot \mathbf{I}_{n, l, m}^{*}\left(\mathbf{e}_{x}+i \mathbf{e}_{y}\right)\right\}\right. \\
& \left.\quad-i m \mathbf{I}_{n^{\prime}, l, m} \cdot \mathbf{I}_{n, l, m}^{*} \mathbf{e}_{z}\right),
\end{aligned}
$$

where $G_{l}\left(q_{n}\right)$ and $a_{l, m}$ are defined in (33) and (34), respectively. Evidently, those properties of $\overline{\mathbf{F}}_{S}$ that have been discussed at the end of Sec. 3 are valid for $\overline{\mathbf{N}}_{S}$ of (45) as well.

Note, however, that the time-averaged torque $\overline{\mathbf{N}}_{S}$ around the center of the sphere $\bar{S}$ may be different from zero only under certain conditions. Taking into account that $\mathbf{I}_{n, l, m}=(-1)^{m} \mathbf{I}_{n, l,-m}^{*}$, which follows from (13) and (91), we find that

$$
\begin{aligned}
& \sum_{m=-l}^{+l} \mathbf{I}_{n^{\prime}, l, m} \times \mathbf{I}_{n, l, m}^{*}=-\sum_{m=-l}^{+l} \mathbf{I}_{n, l, m} \times \mathbf{I}_{n^{\prime}, l, m}^{*}, \\
& \sum_{m=-l}^{+l} m \mathbf{I}_{n^{\prime}, l, m} \cdot \mathbf{I}_{n, l, m}^{*}=-\sum_{m=-l}^{+l} m \mathbf{I}_{n, l, m} \cdot \mathbf{I}_{n^{\prime}, l, m}^{*} .
\end{aligned}
$$


Furthermore, taking the definition (34) of $a_{l, m}$ into account, we have

$$
\begin{aligned}
\sum_{m=-l}^{+l} \frac{a_{l, m+1}}{a_{l, m}}(l-m) \mathbf{I}_{n^{\prime}, l, m+1} \cdot \mathbf{I}_{n, l, m}^{*} & =-\sum_{m=-l}^{l-1} \frac{a_{l, m+1}}{a_{l, m}}(l-m) \mathbf{I}_{n^{\prime}, l,-m-1}^{*} \cdot \mathbf{I}_{n, l,-m} \\
& =-\sum_{m=-l}^{l-1} \frac{a_{l,-m}}{a_{l,-m-1}}(l+m+1) \mathbf{I}_{n^{\prime}, l, m}^{*} \cdot \mathbf{I}_{n, l, m+1} \\
& =-\sum_{m=-l}^{+l} \frac{a_{l, m+1}}{a_{l, m}}(l-m) \mathbf{I}_{n, l, m+1} \cdot \mathbf{I}_{n^{\prime}, l, m}^{*} .
\end{aligned}
$$

Consequently, the $n, n^{\prime}$-sums over these terms on the right-hand side of (45) and thus $\overline{\mathbf{N}}_{S}$ vanish, unless there are at least two external current distributions, indicated, e.g., by $n=1$ and $n=2$, having the same frequency $\omega_{1}=\omega_{2}$ (which implies that $q_{1}=q_{2}$, see (15)) and a phase difference $\alpha_{1}-\alpha_{2} \neq 0, \pm \pi$.

5. Change of the coordinate system. The time-averaged power absorption (16), force (32), and torque (45), are functionals of the external current density fields $\mathbf{j}_{n}(\mathbf{x})$ via the coefficients

$$
\mathbf{I}_{n, l, m}=\frac{1}{a_{l, m}} \int_{\mathbb{R}^{3} \backslash S^{+}} X_{l}^{m^{*}}(\mathbf{x}) \mathbf{j}_{n}(\mathbf{x}) d^{3} x .
$$

As already pointed out at the end of Sec. 3 , the functions $\mathbf{j}_{n}(\mathbf{x})$ are defined in a frame of reference the origin of which is fixed at the center of the sphere $\bar{S}$ (see, e.g., Fig. 1). A movement of the sphere relative to the external current distributions consequently affects $\mathbf{I}_{n, l, m}$ and the related quantities in the form of a coordinate transformation of $\mathbf{j}_{n}(\mathbf{x})$. From a practical point of view, such dependence is, however, very inconvenient, because in the present case it requires recalculations of the integrals of (48). Therefore, we investigate in this section the possibility of expressing the coefficients $\mathbf{I}_{n, l, m}$ and hence the related results like power, force, and torque in a displaced "laboratory" coordinate system that may no longer be attached to the sphere $\bar{S}$, so that by this means a movement of the sphere no longer influences these integrals.

According to Fig. 2 on p. 506, let $(x, y, z)$ be, as before, the coordinates of a point $\mathbf{x}$ in a frame of reference fixed at the center of the sphere and $(\hat{x}, \hat{y}, \hat{z})$ those of a point $\hat{\mathbf{x}}$ in a displaced (not rotated) laboratory frame of reference. Denoting in the laboratory coordinate system the position vector of the center of $\bar{S}$ by $\mathbf{x}_{S}$ and the external current density fields by $\hat{\mathbf{j}}_{n}(\hat{\mathbf{x}})$, we find the relations

$$
\hat{\mathbf{x}}=\mathbf{x}+\mathbf{x}_{S}, \quad \mathbf{j}_{n}(\mathbf{x}) \equiv \mathbf{j}_{n}\left(\hat{\mathbf{x}}-\mathbf{x}_{S}\right)=: \hat{\mathbf{j}}_{n}(\hat{\mathbf{x}}),
$$

and thus for $(48)$

$$
\mathbf{I}_{n, l, m}=\frac{1}{a_{l, m}} \int_{\mathbb{R}^{3} \backslash S^{+}} X_{l}^{m^{*}}\left(\hat{\mathbf{x}}-\mathbf{x}_{S}\right) \hat{\mathbf{j}}_{n}(\hat{\mathbf{x}}) d^{3} \hat{x}
$$




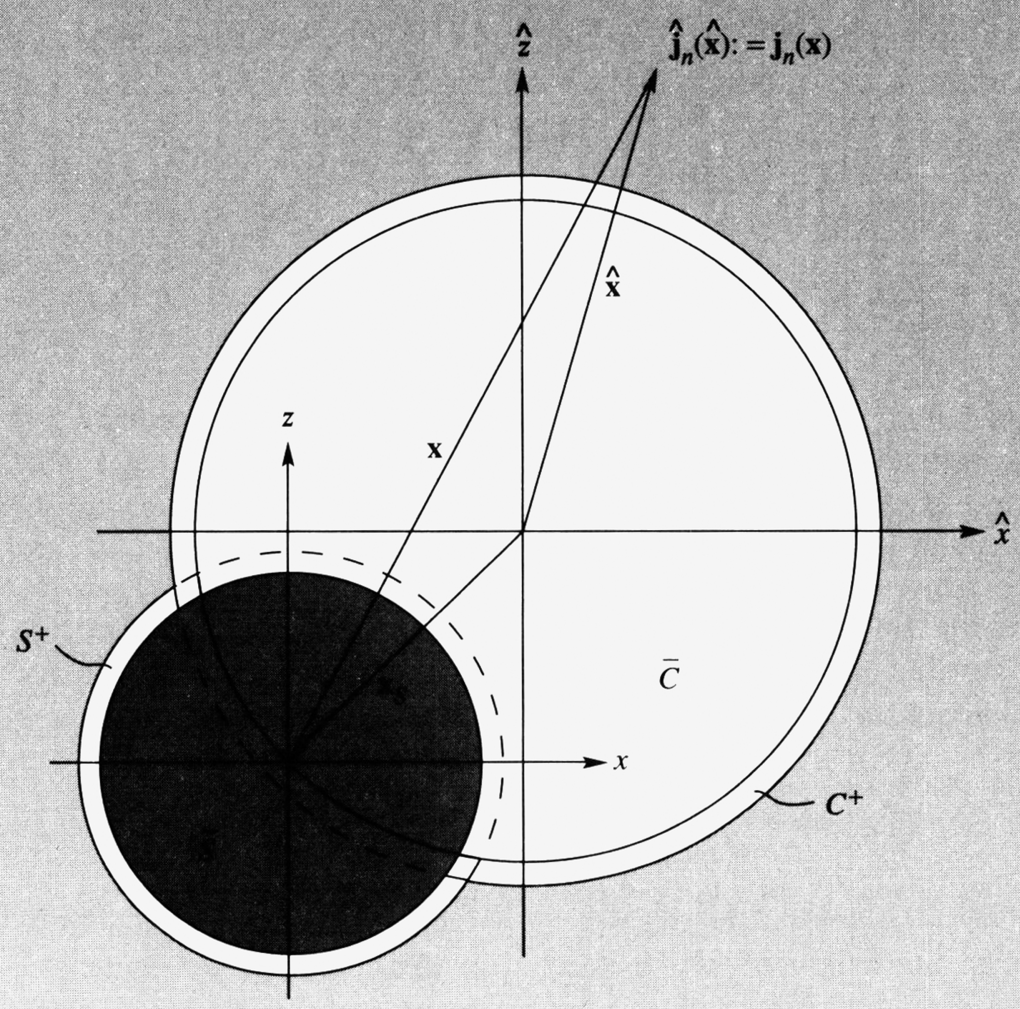

FIG. 2. Sketch of the metal sphere $\bar{S}$, and the $(x, y, z)$ coordinate system shifted by the vector $\mathbf{x}_{S}$ from the center of the laboratory $(\hat{x}, \hat{y}, \hat{z})$ frame of reference. The given external current density distribution is assumed to be different from zero outside of $C^{+} \cup S^{+}$ only.

To achieve our goal we expand the function $X_{l}^{m}\left(\hat{\mathbf{x}}-\mathbf{x}_{S}\right)$ in a power series around $\hat{\mathbf{x}}$. When the properties of $X_{l}^{m}$, derived in Appendix B, are considered, this is, however, an easy and straightforward task that can be reduced to the series representation (107) of $\left|\mathbf{x}-\mathbf{x}^{\prime}\right|^{-1}$. In order to formulate convergence conditions for this procedure let us at first define two additional spherical point sets

$$
\bar{C}:=\left\{\hat{\mathbf{x}}:|\hat{\mathbf{x}}| \leq r_{S}\right\}, \quad C^{+}:=\left\{\hat{\mathbf{x}}:|\hat{\mathbf{x}}|<R_{C^{+}}, R_{C^{+}}>r_{S}\right\}
$$

of radius $r_{S} \equiv\left|\mathbf{x}_{S}\right|$ and $R_{C^{+}}$, respectively, and centered in the origin of the laboratory (hat) coordinate system; see Fig. 2. The definition of $\bar{C}$ implies that $\mathbf{x}_{S} \in \partial \bar{C}$, i.e., the center of $\bar{S}$ lies on the boundary of $\bar{C}$. Taking (94), (95), and (92) into account, we then find that for fixed $\mathbf{x}_{S}$ in the case of $m \geq 0$

$$
X_{l}^{m}\left(\hat{\mathbf{x}}-\mathbf{x}_{S}\right)=2^{m / 2} \frac{\partial^{l-m}}{\partial \hat{z}^{l-m}} \frac{\partial^{m}}{\partial \hat{\eta}^{m}} X_{0}^{0}\left(\hat{\mathbf{x}}-\mathbf{x}_{S}\right)=2^{m / 2} \frac{\partial^{l-m}}{\partial \hat{z}^{l-m}} \frac{\partial^{m}}{\partial \hat{\eta}^{m}} \frac{1}{\left|\hat{\mathbf{x}}-\mathbf{x}_{S}\right|} .
$$


Supposing that $\hat{\mathbf{x}} \in \mathbb{R}^{3} \backslash C^{+}$, the last term can be substituted by (107) leading to

$$
\begin{aligned}
X_{l}^{m}\left(\hat{\mathbf{x}}-\mathbf{x}_{S}\right) & =\sum_{\lambda=0}^{\infty} \sum_{\mu=-\lambda}^{+\lambda} \frac{4 \pi}{(2 \lambda+1) a_{\lambda, \mu}} r_{S}^{\lambda} Y_{\lambda}^{\mu^{*}}\left(u_{S}, \varphi_{S}\right) 2^{m / 2} \frac{\partial^{l-m}}{\partial \hat{z}^{l-m}} \frac{\partial^{m}}{\partial \hat{\eta}^{m}} X_{\lambda}^{\mu}(\hat{\mathbf{x}}) \\
& =\sum_{\lambda=0}^{\infty} \sum_{\mu=-\lambda}^{+\lambda} \frac{4 \pi}{(2 \lambda+1) a_{\lambda, \mu}} r_{S}^{\lambda} Y_{\lambda}^{\mu^{*}}\left(u_{S}, \varphi_{S}\right) X_{\lambda+l}^{\mu+m}(\hat{\mathbf{x}}),
\end{aligned}
$$

where $\left(r_{S}, u_{S}, \varphi_{S}\right)$ with $u_{S}:=\cos \theta_{S}$ denote the spherical coordinates of $\mathbf{x}_{S}$. A justification for the term-by-term differentiation of the series is given in Appendix C. Equation (53) holds for $m<0$ too. Because of the symmetry properties (90), (91), and (106) of $a_{l, m}, X_{l}^{m}$, and $Y_{l}^{m}$, the proof of this case can immediately be reduced to that of $m \geq 0$.

Insertion of (53) in (50) and term-by-term integration finally results for all $l, m \in$ $\mathbb{Z}$ with $|m| \leq l$ in

$$
\mathbf{I}_{n, l, m}=\sum_{\lambda=0}^{\infty} \sum_{\mu=-\lambda}^{+\lambda} \frac{4 \pi}{2 \lambda+1} r_{S}^{\lambda} Y_{\lambda}^{\mu}\left(u_{S}, \varphi_{S}\right) \frac{a_{\lambda+l, \mu+m}}{a_{l, m} a_{\lambda, \mu}} \widehat{\mathbf{I}}_{n, \lambda+l, \mu+m},
$$

where in analogy to (13)

$$
\begin{aligned}
\widehat{\mathbf{I}}_{n, l, m} & :=\frac{1}{a_{l, m}} \int_{\mathbb{R}^{3} \backslash S^{+} \backslash C^{+}} X_{l}^{m^{*}}(\mathbf{x}) \hat{\mathbf{j}}_{n}(\mathbf{x}) d^{3} x \\
& \equiv \int_{\mathbb{R}^{3} \backslash S^{+} \backslash C^{+}} \hat{\mathbf{j}}_{n}(r, u, \varphi) r^{-l+1} Y_{l}^{m^{*}}(u, \varphi) d \varphi d u d r .
\end{aligned}
$$

Provided the laboratory (hat) coordinate system is not attached to the sphere $\bar{S}$, the functions $r_{S}^{\lambda} Y_{\lambda}^{u}\left(u_{S}, \varphi_{S}\right)$ in (54) describe explicitly the dependence of $\mathbf{I}_{n, l, m}$, and therefore also of $\bar{P}_{S}, \overline{\mathbf{F}}_{S}$, and $\overline{\mathbf{N}}_{S}$, on the position of $\bar{S}$. Moreover, as long as the external current density fields are fixed to the laboratory coordinate system, its functional representations in this frame of reference, i.e., $\hat{\mathbf{j}}_{n}(\hat{\mathbf{x}})$, and therefore also the integrals $\widehat{\mathbf{I}}_{n, l, m}$ remain unchanged under motions of $\bar{S}$.

We found, see Appendix $\mathrm{C}$, that (53) converges uniformly for $\hat{\mathbf{x}} \notin C^{+}$. Consequently, term-by-term integration leading to (54) is permitted, supposing that $\hat{\mathbf{j}}_{n}$ is integrable, if $\hat{\mathbf{j}}_{n}(\hat{\mathbf{x}}) \equiv \mathbf{0}$ for all $\hat{\mathbf{x}} \in C^{+}$. Hence we conclude that, due to (5), the series expressions for power absorption, force, and torque in (16), (32), and (45) converge in connection with the series representation for $\mathbf{I}_{n, l, m}$ in $(54)$, if $\hat{\mathbf{j}}_{n}(\hat{\mathbf{x}}) \equiv \mathbf{0}$ for all $\hat{\mathbf{x}} \in S^{+} \cup C^{+}$. This means that the given set of external current density fields has to be located completely outside of the area bordered by the bold line in Fig. 2. Evidently, the dimension of this area and hence the variation of the sphere $\bar{S}$ depends on the choice of the origin of the laboratory (hat) frame of reference.

Collecting equal orders of $r_{S}$, the coefficient products appearing in $\bar{P}_{S}, \overline{\mathbf{F}}_{S}$, and $\overline{\mathbf{N}}_{S}$ then read at full length

$$
\frac{a_{l+l_{0}, m+m_{0}}}{a_{l, m}}\left(\begin{array}{c}
\mathbf{I}_{n^{\prime}, l+l_{0}, m+m_{0}} \cdot \mathbf{I}_{n, l, m}^{*} \\
\mathbf{I}_{n^{\prime}, l+l_{0}, m+m_{0}} \times \mathbf{I}_{n, l, m}^{*}
\end{array}\right)=\sum_{\lambda=0}^{\infty} r_{S}^{\lambda}\left(\begin{array}{l}
m_{0} l_{0} C_{n, n^{\prime}}^{\lambda, l, m}\left(u_{S}, \varphi_{S}\right) \\
m_{0} \mathbf{C}_{n, n^{\prime}}^{\lambda, l, m}\left(u_{S}, \varphi_{S}\right)
\end{array}\right),
$$


where

$$
\begin{aligned}
& \left(\begin{array}{l}
{ }_{l_{0}}^{m_{0}} C_{n, n^{\prime}}^{\lambda, l, m}\left(u_{S}, \varphi_{S}\right) \\
m_{0} \mathbf{C}_{n, n^{\prime}}^{\lambda, l, m}\left(u_{S}, \varphi_{S}\right)
\end{array}\right) \\
& :=\sum_{l_{0}^{\prime}=0}^{i} \sum_{\mu^{\prime}=-\lambda^{\prime}}^{+\lambda} \sum_{\mu=-\left(\lambda-\lambda^{\prime}\right)}^{\lambda-\lambda^{\prime}} \frac{16 \pi^{2}}{\left(2 \lambda^{\prime}+1\right)\left(2\left(\lambda-\lambda^{\prime}\right)+1\right)} Y_{\lambda^{\prime}}^{\mu^{\prime}}\left(u_{S}, \varphi_{S}\right) Y_{\lambda-\lambda^{\prime}}^{\mu^{*}}\left(u_{S}, \varphi_{S}\right) \\
& \quad \times \frac{a_{\lambda^{\prime}+l+l_{0}, \mu^{\prime}+m+m_{0}} a_{\lambda-\lambda^{\prime}+l, \mu+m}}{a_{l, m}^{2} a_{\lambda^{\prime}, \mu^{\prime}} a_{\lambda-\lambda^{\prime}, \mu}}\left(\begin{array}{c}
\widehat{\mathbf{I}}_{n^{\prime}, \lambda^{\prime}+l+l_{0}, \mu^{\prime}+m+m_{0}} \cdot \widehat{\mathbf{I}}_{\lambda, \lambda-\lambda^{\prime}+l, \mu+m}^{*} \\
\widehat{\mathbf{I}}_{n^{\prime}, \lambda^{\prime}+l+l_{0}, \mu^{\prime}+m+m_{0}} \times \widehat{\mathbf{I}}_{n, \lambda-\lambda^{\prime}+l, \mu+m}^{*}
\end{array}\right) .
\end{aligned}
$$

From the definition (55), or more clearly from (63) together with (65) we find, for the order of the coefficients, $\left|\widehat{\mathbf{I}}_{n, l, m}\right|=O\left(1 / R^{l}\right)$, where $R:=\max \left\{R_{C^{+}}, R_{S^{+}}\right\}$. This again implies that ${ }_{l_{0}}^{m_{0}} C_{n, n^{\prime}}^{\lambda, l, m}=O\left(1 / R^{\lambda+2 l+l_{0}}\right)$.

After insertion of (56) the time-averaged heating power (16), the time-averaged force (32), and the time-averaged torque (45) of the sphere $\bar{S}$ in a laboratory (hat) frame of reference can be expressed as

$$
\begin{aligned}
\bar{P}_{S}\left(r_{S}, u_{S}, \varphi_{S}\right)= & \frac{1}{2 R_{S} \sigma_{S}} \sum_{n, n^{\prime}} \sum_{l=0}^{\infty} \sum_{\lambda=0}^{\infty} \delta_{\omega_{n}, \omega_{n^{\prime}}} H_{l}\left(q_{n}\right) \cos \left(\alpha_{n}-\alpha_{n^{\prime}}\right) R_{S}^{2 l} r_{S}^{\lambda} \\
& \times \sum_{m=-l}^{+l}{ }_{0}^{0} C_{n, n^{\prime}}^{\lambda, l, m}\left(u_{S}, \varphi_{S}\right), \\
\overline{\mathbf{F}}_{S}\left(r_{S}, u_{S}, \varphi_{S}\right)= & \frac{\mu_{0}}{2} \sum_{n, n^{\prime}} \sum_{l=0}^{\infty} \sum_{\lambda=0}^{\infty} \delta_{\omega_{n}, \omega_{n^{\prime}}} \operatorname{Re}\left\{G_{l}\left(q_{n}\right) e^{-i\left(\alpha_{n}-\alpha_{n^{\prime}}\right)}\right\} R_{S}^{2 l+1} r_{S}^{\lambda} \\
& \times \sum_{m=-l}^{+l}\left(\operatorname{Re}\left\{{ }_{1}^{1} C_{n, n^{\prime}}^{\lambda, l}\left(u_{S}, \varphi_{S}\right)\left(\mathbf{e}_{x}+i \mathbf{e}_{y}\right)\right\}+{ }_{1}^{0} C_{n, n^{\prime}}^{\lambda, l, m}\left(u_{S}, \varphi_{S}\right) \mathbf{e}_{z}\right), \\
\overline{\mathbf{N}}_{S}\left(r_{S}, u_{S}, \varphi_{S}\right)= & \frac{\mu_{0}}{2} \sum_{n, n^{\prime}} \sum_{i=0}^{\infty} \sum_{\lambda=0}^{\infty} \delta_{\omega_{n}, \omega_{n^{\prime}}} \operatorname{Re}\left\{G_{l}\left(q_{n}\right) e^{-i\left(\alpha_{n}-\alpha_{n^{\prime}}\right)}\right\} R_{S}^{2 l+1} r_{S}^{\lambda} \\
& \times \sum_{m=-l}^{+l}\left({ }_{0}^{0} \mathbf{C}_{n, n^{\prime}}^{\lambda, l, m}\left(u_{S}, \varphi_{S}\right)-(l-m) \operatorname{Im}\left\{{ }_{0}^{1} C_{n, n^{\prime}}^{\lambda, l, m}\left(u_{S}, \varphi_{S}\right)\left(\mathbf{e}_{x}+i \mathbf{e}_{y}\right)\right\}\right. \\
& \left.\quad-i m_{0}^{0} C_{n, n^{\prime}}^{\lambda, l, m}\left(u_{S}, \varphi_{S}\right) \mathbf{e}_{z}\right) .
\end{aligned}
$$

In most practical cases the series over $l$ and $\lambda$ converge quickly enough to neglect all but the first nonvanishing terms. Moreover, with the help of (59), it is evidently an easy task to derive sufficient conditions for the local stability of the spherical sample $\bar{S}$ close to an equilibrium point of the magnetic force field. 


\section{Special cases.}

6.1. Azimuthal external current distribution. In this section we treat the special case of azimuthal external currents, which is often encountered in practice. This means that the set of external current density distributions (5) has rotational symmetry, and, moreover, that the current directions have no component radial or parallel to the axis of symmetry. In a laboratory frame of reference whose $\hat{z}$-axis is identified with the axis of symmetry this special case can be written as

$$
\hat{\mathbf{j}}_{n}(\hat{\mathbf{x}})=\hat{j}_{n}(\hat{r}, \hat{u}) \mathbf{e}_{\varphi}(\hat{\varphi}),
$$

where

$$
\mathbf{e}_{\varphi}(\hat{\varphi})=-\sin \hat{\varphi} \mathbf{e}_{x}+\cos \hat{\varphi} \mathbf{e}_{y}
$$

is the unit vector in the azimuthal direction. The scalar function $\hat{j}_{n}(\hat{r}, \hat{u})$ describes the remaining $\hat{r}$ and $\hat{u}(\hat{u}:=\cos \hat{\theta})$ dependence of the $n$th external current density field. In $[9$, Sec. 5] it has been shown that for this case with the notation of (84)

$$
\widehat{\mathbf{I}}_{n, l, m}=-i \sqrt{\frac{\pi}{2}} \widehat{I}_{n, l}\left[\delta_{m, 1} \mathbf{e}_{\eta}^{*}+\delta_{m,-1} \mathbf{e}_{\eta}\right]
$$

where

$$
\hat{I}_{n, l}:=\sqrt{\frac{2 l+1}{l(l+1)}} \int_{R_{C^{+}}}^{\infty} \int_{-1}^{+1} \hat{j}_{n}(r, u) r^{-l+1} P_{l}^{1}(u) d u d r .
$$

If, moreover, the external current density distributions $\hat{j}_{n}(\hat{r}, \hat{u})$ are approximated by a set of circular current loops, Eq. (64) modifies to [9, Sec. 5]

$$
\widehat{I}_{n, l}=\sqrt{\frac{2 l+1}{l(l+1)}} I_{n} \hat{r}_{n}^{-l} \sin \hat{\theta}_{n} P_{l}^{1}\left(\cos \hat{\theta}_{n}\right)
$$

where $\hat{r}_{n}$ and $\hat{\theta}_{n}$ are the coordinates of the $n$th current loop carrying the current $I_{n}$ of frequency $\omega_{n}$ and phase $\alpha_{n}$.

Using (63) and considering the properties (85), (90), and (106), we find that the coefficients (57) read

$$
\begin{aligned}
{ }_{l_{0}}^{m_{0}} C_{n, n^{\prime}}^{\lambda, l, m}\left(u_{S}, \varphi_{S}\right)= & \frac{\pi}{2} \sum_{\lambda^{\prime}=0}^{\lambda} \frac{16 \pi^{2} \widehat{I}_{n^{\prime}, \lambda^{\prime}+l+l_{0}} \widehat{I}_{n, \lambda-\lambda^{\prime}+l}}{\left(2 \lambda^{\prime}+1\right)\left(2\left(\lambda-\lambda^{\prime}\right)+1\right)} \frac{a_{\lambda^{\prime}+l+l_{0}, 1} a_{\lambda-\lambda^{\prime}+l, 1}}{a_{l, m}^{2}} \\
\times & {\left[\frac{Y_{\lambda^{\prime}}^{l-m-m_{0}}\left(u_{S}, \varphi_{S}\right) Y_{\lambda-\lambda^{\prime}}^{l-m^{*}}\left(u_{S}, \varphi_{S}\right)}{a_{\lambda^{\prime}, 1-m-m_{0}} a_{\lambda-\lambda^{\prime}, 1-m}}\right.} \\
& \left.+(-1)^{m_{0}} \frac{Y_{\lambda^{\prime}}^{1+m+m_{0}^{*}}\left(u_{S}, \varphi_{S}\right) Y_{\lambda-\lambda^{\prime}}^{1+m}\left(u_{S}, \varphi_{S}\right)}{a_{\lambda^{\prime}, 1+m+m_{0}} a_{\lambda-\lambda^{\prime}, 1+m}}\right],
\end{aligned}
$$




$$
\begin{aligned}
{ }_{l_{0}}^{m_{0}} \mathbf{C}_{n, n^{\prime}}^{\lambda, l, m}\left(u_{S}, \varphi_{S}\right)= & \frac{\pi}{2} \sum_{\lambda^{\prime}=0}^{\lambda} \frac{16 \pi^{2} \widehat{I}_{n^{\prime}, \lambda^{\prime}+l+l_{0}} \widehat{I}_{n, \lambda-\lambda^{\prime}+l}}{\left(2 \lambda^{\prime}+1\right)\left(2\left(\lambda-\lambda^{\prime}\right)+1\right)} \frac{a_{\lambda^{\prime}+l+l_{0}, 1} a_{\lambda-\lambda^{\prime}+l, 1}}{a_{l, m}^{2}} \\
& \times\left[\frac{Y_{\lambda^{\prime}}^{1-m-m_{0}}\left(u_{S}, \varphi_{S}\right) Y_{\lambda-\lambda^{\prime}}^{1-m^{*}}\left(u_{S}, \varphi_{S}\right)}{a_{\lambda^{\prime}, 1-m-m_{0}} a_{\lambda-\lambda^{\prime}, 1-m}}\right. \\
& \left.-(-1)^{m_{0}} \frac{Y_{\lambda^{\prime}}^{1+m+m_{0}^{*}}\left(u_{S}, \varphi_{S}\right) Y_{\lambda-\lambda^{\prime}}^{1+m}\left(u_{S}, \varphi_{S}\right)}{a_{\lambda^{\prime}, 1+m+m_{0}} a_{\lambda-\lambda^{\prime}, 1+m}}\right] i \mathbf{e}_{z} .
\end{aligned}
$$

According to (103), both terms in the brackets of (66) and (67) are real for $m_{0}=0$. Hence, in this case, the coefficients have the properties

$$
{ }_{l_{0}}^{0} \mathbf{C}_{n, n^{\prime}}^{\lambda, l,-m}\left(u_{S}, \varphi_{S}\right)=-{ }_{l_{0}}^{0} \mathbf{C}_{n, n^{\prime}}^{\lambda, l, m}\left(u_{S}, \varphi_{S}\right)
$$

and

$$
{ }_{l_{0}}^{0} C_{n, n^{\prime}}^{\lambda, l,-m}\left(u_{S}, \varphi_{S}\right)={ }_{l_{0}}^{0} C_{n, n^{\prime}}^{\lambda, l, m}\left(u_{S}, \varphi_{S}\right) .
$$

When inserted in (58), (59), or (60), the above results finally yield the time-averaged power, force, or torque of a conducting sphere in an azimuthal external current density field. In particular, (68a) and (68b) imply that the summations over $m$ of the first and third terms on the right-hand side of (60) identically disappear. Consequently there is no component of the torque through the center of $\bar{S}$ parallel to the axis of symmetry ( $\hat{z}$-axis). Note, that due to (103), i.e., $Y_{l}^{m} \equiv 0$ for $l<|m|$, an appreciable number of coefficients ${ }_{l_{0}}^{m_{0}} C_{n, n^{\prime}}^{\lambda, l, m}$ in these equations become zero.

6.2. Azimuthal and mirror-symmetric external current distributions. Azimuthal external current distributions with additional mirror symmetry are used in some electromagnetic levitation facilities working under microgravity conditions [1], [2]. Here, two different azimuthal current density fields of different frequencies are superposed, where one, indicated by $n=1$, has an additional mirror symmetry with respect to a plane perpendicular to the $\hat{z}$-axis and the other one, indicated by $n=2$, has an additional anti-mirror symmetry, cf. Fig. 3. Provided the mirror plane is defined by $\hat{z}=0$, this means that for $n=1$,

$$
\hat{j}_{1}(\hat{r},-\hat{u})=\hat{j}_{1}(\hat{r}, \hat{u})
$$

and for $n=2$,

$$
\hat{j}_{2}(\hat{r},-\hat{u})=-\hat{j}_{2}(\hat{r}, \hat{u}) \text {. }
$$

Due to the different frequencies, the occurrence of $\delta_{\omega_{n}, \omega_{n^{\prime}}}$ in (58) and (59) allows for each of the two current density fields a separate calculation of $\bar{P}_{S}$ and $\overline{\mathbf{F}}_{S}$. From the discussion at the end of Sec. 4 we immediately conclude that $\overline{\mathbf{N}}_{S} \equiv \mathbf{0}$ in both cases. 


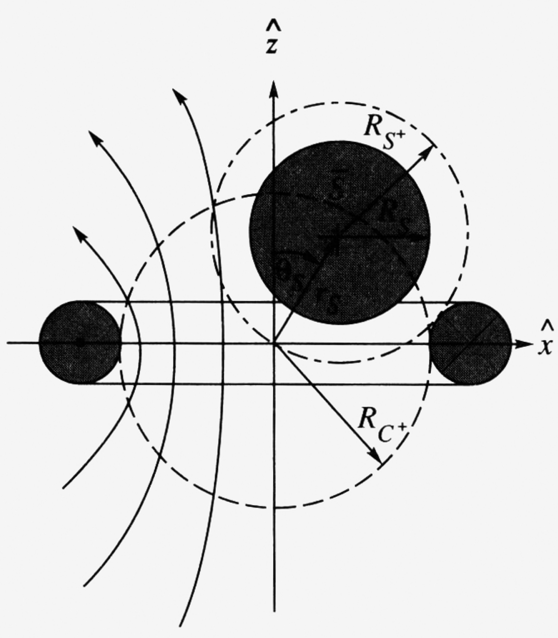

(a)

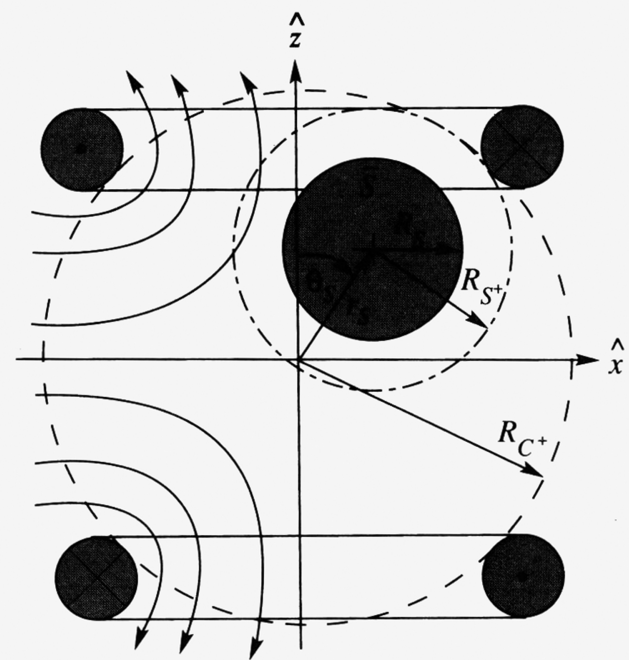

(b)

FIG. 3. Schematic cross-sectional drawing of the spherical sample $\bar{S}$ surrounded by (a) an azimuthal, mirror-symmetric external current density distribution, here exemplified by a single toroidal current coil; (b) an azimuthal, anti-mirror-symmetric external current density distribution, here exemplified by a pair of toroidal coils carrying oppositely directed currents. A part of the corresponding magnetic field lines is sketched too. The results derived in Sec. 6 are valid only if the center of $\bar{S}$ remains inside of the dash-lined sphere of convergence, i.e., if $r_{S}<R_{C^{+}}$.

1. Mirror-symmetric current density. First of all we calculate for the $n=1$ current density field (69), cf. Fig. 3(a), an explicit expression for $\bar{P}_{S}$ and $\overline{\mathbf{F}}_{S}$ in the first two nonvanishing orders of the sample distance $r_{S}$ and the sample radius $R_{S}$. Since $P_{l}^{1}(-u)=(-1)^{l+1} P_{l}^{1}(u)$ (see, e.g., [13]), we find from (64) that $\widehat{I}_{1, l} \neq 0$ only if $l=1,3,5, \ldots$. Hence it follows from (66) that ${ }_{l_{0}}^{m_{0}} C_{1,1}^{\lambda, l, m} \neq 0$ only if $\lambda+2 l+l_{0}=2,4,6, \ldots$, and if $\lambda^{\prime}+l+l_{0}=1,3,5, \ldots$ for any $\lambda^{\prime}$ with $0 \leq \lambda^{\prime} \leq \lambda$. Moreover, because of (103), there are only a few values of $m$ for which (66) is different from zero. Hence, in the present case, we find using (68b), (103), and the definition of $P_{l}^{m}(u)$ from [13] that the time-averaged power absorbed by the sphere $\bar{S}$ is

$$
\begin{aligned}
\bar{P}_{S}\left(r_{S}, u_{S}, \varphi_{S}\right)=\frac{\pi}{2 R_{S} \sigma_{S}}[ & R_{S}^{2} H_{1} \widehat{I}_{1,1}^{2}+R_{S}^{6} H_{3} \widehat{I}_{1,3}^{2}+O\left(R_{S}^{10} / R_{S^{+}}^{10}\right) \\
& +r_{S}^{2}\left(H_{0} \frac{\widehat{I}_{1,1}^{2}}{6} \sin ^{2} \theta_{S}+R_{S}^{2} H_{1} \sqrt{\frac{18}{7}} \widehat{I}_{1,1} \widehat{I}_{1,3}\left(3 \cos ^{2} \theta_{S}-1\right)\right. \\
& \left.\left.+O\left(R_{S}^{4} /\left(R_{S^{+}}^{4} R_{C^{+}}^{2}\right)\right)\right)+O\left(r_{S}^{4} / R_{C^{+}}^{4}\right)\right]
\end{aligned}
$$


where the function $H_{l}\left(q_{1}\right)$ of (17) has been abbreviated by $H_{l}$. For an explanation of the geometrical quantities, see Fig. 3.

The time-averaged force exerted on $\bar{S}$ can be represented in the form

$$
\overline{\mathbf{F}}_{S}\left(r_{S}, u_{S}, \varphi_{S}\right)=F_{\rho}\left(r_{S}, u_{S}\right)\left(\cos \varphi_{S} \mathbf{e}_{x}+\sin \varphi_{S} \mathbf{e}_{y}\right)+F_{z}\left(r_{S}, u_{S}\right) \mathbf{e}_{z}
$$

with

$$
\begin{array}{r}
F_{\rho}\left(r_{S}, u_{S}\right)=-\frac{\mu_{0} \pi}{2}\left[r_{S} \sin \theta_{S}\left(R_{S} G_{0} \frac{\widehat{I}_{1,1}^{2}}{6}-R_{S}^{3} G_{1} \sqrt{\frac{18}{7}} \widehat{I}_{1,1} \widehat{I}_{1,3}+O\left(\frac{R_{S}^{5}}{R_{S^{+}}^{5} R_{C^{+}}}\right)\right)\right. \\
+r_{S}^{3} \sin \theta_{S}\left(R_{S} G_{0} \frac{1}{\sqrt{14}} \widehat{I}_{1,1} \widehat{I}_{1,3}\left(3 \cos ^{2} \theta_{S}-1\right)\right. \\
+R_{S}^{3} G_{1}\left[\frac{9}{28} \widehat{I}_{1,3}^{2}\left(5+3 \cos ^{2} \theta_{S}\right)\right. \\
\left.-\sqrt{\frac{405}{176}} \widehat{I}_{1,1} \widehat{I}_{1,5}\left(5 \cos ^{2} \theta_{S}-1\right)\right] \\
\left.\left.+O\left(\frac{R_{S}^{5}}{R_{S^{+}}^{5} R_{C^{+}}^{3}}\right)\right)+O\left(r_{S}^{5} / R_{C^{+}}^{5}\right)\right],
\end{array}
$$

and

$$
\begin{array}{r}
F_{z}\left(r_{S}, u_{S}\right)=-\frac{\mu_{0} \pi}{2}\left[r _ { S } \operatorname { c o s } \theta _ { S } \left(R_{S}^{3} G_{1} \sqrt{\frac{72}{7}} \widehat{I}_{1,1} \widehat{I}_{1,3}\right.\right. \\
+R_{S}^{3} \cos \theta_{S}\left(R_{S} G_{0} \sqrt{\frac{2}{7}} \widehat{I}_{1,1} \widehat{I}_{1,3} \widehat{I}_{1,3}^{2}+O\left(\frac{R_{S}^{7}}{R_{S^{+}}^{7} R_{C^{+}}}\right)\right) \\
+R_{S}^{3} G_{1}\left[\frac{18}{7} \widehat{I}_{1,3}^{2}\left(1+\cos ^{2} \theta_{S}\right)\right. \\
+\sqrt{\left.\frac{180}{11} \widehat{I}_{1,1} \widehat{I}_{1,5}\left(5 \cos ^{2} \theta_{S}-3\right)\right]} \\
\left.\left.+O\left(\frac{R_{S}^{5}}{R_{S^{+}}^{5} R_{C^{+}}^{3}}\right)\right)+O\left(r_{S}^{5} / R_{C^{+}}^{5}\right)\right] .
\end{array}
$$

Above we used the abbreviation $G_{l}$ for the function $\operatorname{Re}\left\{G_{l}\left(q_{1}\right)\right\}$ of $(33)$.

2. Anti-mirror-symmetric current density. Next, we calculate analogous formulas for the $n=2$ current density field (70); cf. Fig. 3(b). In this case, the symmetry property of $P_{l}^{1}(u)$ implies that $\widehat{I}_{2, l} \neq 0$ only if $l=2,4,6, \ldots$. Hence it follows from (66) that ${ }_{l_{0}}^{m_{0}} C_{2,2}^{\lambda, l, m} \neq 0$ only if $\lambda+2 l+l_{0}=4,6,8, \ldots$, and if $\lambda^{\prime}+l+l_{0}=$ $2,4,6, \ldots$ for any $\lambda^{\prime}$ with $0 \leq \lambda^{\prime} \leq \lambda$. Now using the abbreviations $H_{l}:=H_{l}\left(q_{2}\right)$ 
and $G_{l}:=\operatorname{Re}\left\{G_{l}\left(q_{2}\right)\right\}$, we find in the present case that for the time-averaged power absorbed by the sphere $\bar{S}$

$$
\begin{aligned}
& \bar{P}_{S}\left(r_{S}, u_{S}, \varphi_{S}\right)=\frac{\pi}{2 R_{S} \sigma_{S}}[ R_{S}^{4} H_{2} \widehat{I}_{2,2}^{2} \\
&+r_{S}^{2}\left(R _ { S } ^ { 2 } H _ { 1 } \frac { 9 } { 1 0 } \widehat { I } _ { 2 , 2 } ^ { 2 } \left(1+\widehat{I}_{2,4}^{2}+O\left(R_{S}^{12} / R_{S^{+}}^{12}\right)\right.\right. \\
&+R_{S}^{4} H_{2} \sqrt{\frac{50}{3}} \widehat{I}_{2,2} \widehat{I}_{2,4}\left(3 \cos ^{2} \theta_{S}-1\right) \\
&\left.\left.+O\left(R_{S}^{6} /\left(R_{S^{+}}^{6} R_{C^{+}}^{2}\right)\right)\right)+O\left(r_{S}^{4} / R_{C^{+}}^{4}\right)\right]
\end{aligned}
$$

and for the components of the time-averaged force (72) exerted on $\bar{S}$

$$
\begin{array}{r}
F_{\rho}\left(r_{S}, u_{S}\right)=-\frac{\mu_{0} \pi}{2}[ \\
r_{S} \sin \theta_{S}\left(R_{S}^{3} G_{1} \frac{9}{10} \widehat{I}_{2,2}^{2}-R_{S}^{5} G_{2} \sqrt{\frac{50}{3}} \widehat{I}_{2,2} \widehat{I}_{2,4}+O\left(\frac{R_{S}^{7}}{R_{S^{+}}^{7} R_{C^{+}}}\right)\right) \\
+r_{S}^{3} \sin \theta_{S}\left(R_{S} G_{0} \frac{3}{10} \widehat{I}_{2,2}^{2} \cos ^{2} \theta_{S}-R_{S}^{3} G_{1} \sqrt{\frac{27}{2}} \widehat{I}_{2,2} \widehat{I}_{2,4} \sin ^{2} \theta_{S}\right. \\
\left.\left.+O\left(\frac{R_{S}^{5}}{R_{S^{+}}^{5} R_{C^{+}}^{3}}\right)\right)+O\left(r_{S}^{5} / R_{C^{+}}^{5}\right)\right],
\end{array}
$$

and

$$
\begin{array}{r}
F_{z}\left(r_{S}, u_{S}\right)=-\frac{\mu_{0} \pi}{2}\left[r_{S} \cos \theta_{S}\left(R_{S}^{3} G_{1} \frac{9}{5} \widehat{I}_{2,2}^{2}+R_{S}^{5} G_{2} \sqrt{\frac{200}{3}} \widehat{I}_{2,2} \widehat{I}_{2,4}+O\left(\frac{R_{S}^{7}}{R_{S^{+}}^{7} R_{C^{+}}}\right)\right)\right. \\
+r_{S}^{3} \cos \theta_{S}\left(R_{S} G_{0} \frac{3}{10} \widehat{I}_{2,2}^{2} \sin ^{2} \theta_{S}\right. \\
\left.+R_{S}^{3} G_{1} \sqrt{96} \widehat{I}_{2,2} \widehat{I}_{2,4} \cos ^{2} \theta_{S}+O\left(\frac{R_{S}^{5}}{R_{S^{+}}^{5} R_{C^{+}}^{3}}\right)\right) \\
\left.+O\left(r_{S}^{5} / R_{C^{+}}^{5}\right)\right] .
\end{array}
$$

Several conclusions can be drawn from these formulas.

(1) Since the power absorption of the sphere $\bar{S}$ in the center of the mirrorsymmetric external current density field $\hat{j}_{1}(\hat{r}, \hat{u})$ is of the order $\bar{P}_{S}=O\left(R_{S}^{2} / R_{S^{+}}^{2}\right)$, see (71), whereas in the center of the anti-mirror-symmetric external current density field $\hat{j}_{2}(\hat{r}, \hat{u})$ it is of the order $\bar{P}_{S}=O\left(R_{S}^{4} / R_{S^{+}}^{4}\right)$, see (75), the inductive heating capability of the former current density field is usually more efficient than that of the latter one. 
(2) The forces generated from the anti-mirror-symmetric current density field $\hat{j}_{2}(\hat{r}, \hat{u})$ have a stable equilibrium point at the origin of the coordinate system, see (76) and (77), and are of the order $F_{z}, F_{\rho}=O\left(R_{S}^{3} / R_{S^{+}}^{3}\right)$ in its neighborhood. On the contrary, mirror-symmetric external current density fields $\hat{j}_{1}(\hat{r}, \hat{u})$ that are mainly concentrated close to the $(\hat{x}, \hat{y})$-plane (cf. Fig. 3(a)) give rise to unstable force components in the $\hat{z}$-direction (74), because in this case $\widehat{I}_{1,3}<0$. If, however, $\hat{j}_{1}$ is chosen in such a way that the integral $\hat{I}_{1,3}=0$, then $F_{z}$ is near the origin of the order $F_{z}=O\left(R_{S}^{9} / R_{S^{+}}^{9}\right)$ only and therefore usually much lower than the corresponding force component generated by $\hat{j}_{2}$.

The above discussion implies that in electromagnetic levitation facilities, in which external current density fields of these symmetries are simultaneously applied [1], [2], inductive heating and positioning of the metallic sample by mirror and antimirror-symmetric current density fields, respectively, can be performed almost independently of each other.

(3) In most electromagnetic levitation facilities, the axis of symmetry ( $\hat{z}$-axis) of the levitation coil is directed along the gravity vector. Comparing (73) with (74) we remark that the force components generated by a mirror-symmetric external current density field, so e.g. by the single coil winding of Fig. 3(a), behave near the origin like $F_{z} / F_{\rho}=O\left(R_{S}^{2} / R_{S^{+}}^{2}\right)$ and are usually lower than 1 . Hence it follows, however, that levitation would generally be more efficient, if the coil axis were pointed perpendicular to the gravity vector.

Appendix A. The function $G_{l}(q)$. In this appendix we prove that for $q \in \mathbb{R}, q \geq 0$, and $l=0,1,2, \ldots$

$$
G_{l}(q):=\sum_{k=1}^{\infty} \frac{8 q^{4}-i 4 q^{2} x_{l+1 / 2, k}^{2}}{\left(4 q^{4}+x_{l+1 / 2, k}^{4}\right) x_{l+1 / 2, k}^{2}}=-\frac{J_{l+3 / 2}((1+i) q)}{(2 l+1) J_{l-1 / 2}((1+i) q)},
$$

where $x_{l+1 / 2, k}, k \in \mathbb{N}$, are the positive real zeros of $J_{l-1 / 2}(x)$ arranged in ascending order of magnitude.

Proof. For $z \in \mathbb{C}$ and $\nu \in \mathbb{R}$ the Bessel functions satisfy the recurrence relation [12]: $z J_{\nu+1}(z)+z J_{\nu-1}(z)=2 \nu J_{\nu}(z)$. Furthermore, if $\nu \neq-1,-2,-3, \ldots$, an asymptotic approximation for $z \rightarrow 0$ is given by: $J_{\nu}(z) \sim\left(\frac{1}{2} z\right)^{\nu} / \Gamma(\nu+1)$. Hence,

$$
-\frac{J_{l+3 / 2}(z)}{(2 l+1) J_{l-1 / 2}(z)}=-\frac{J_{l+1 / 2}(z)}{z J_{l-1 / 2}(z)}+\frac{1}{2 l+1}=-\frac{J_{l+1 / 2}(z)}{z J_{l-1 / 2}(z)}+\lim _{z \rightarrow 0} \frac{J_{l+1 / 2}(z)}{z J_{l-1 / 2}(z)} \text {. }
$$

In $\left[9\right.$, Appendix B] it is proved that for $z \notin \mathbb{R}^{-}$and $z \neq x_{l+1 / 2, k}$

$$
-\frac{J_{l+1 / 2}(z)}{z J_{l-1 / 2}(z)}=\sum_{k=1}^{\infty} \frac{2}{z^{2}-x_{l+1 / 2, k}^{2}}
$$


Inserted into (79) this result implies that

$$
-\frac{J_{l+3 / 2}(z)}{(2 l+1) J_{l-1 / 2}(z)}=\sum_{k=1}^{\infty} \frac{2 z^{2}}{\left(z^{2}-x_{l+1 / 2, k}^{2}\right) x_{l+1 / 2, k}^{2}} .
$$

For $z=(1+i) q$ this equation is identical to (78).

Appendix B. Definition and properties of the function $X_{l}^{m}(\mathbf{x})$. We first introduce the complex-valued differential operator

$$
\frac{\partial}{\partial \eta}:=\frac{1}{\sqrt{2}}\left\{\frac{\partial}{\partial x}+i \frac{\partial}{\partial y}\right\} \text {. }
$$

Using the well-known expressions for the differential operators $\partial / \partial x, \partial / \partial y$, and $\partial / \partial z$ in spherical coordinates $(r, u, \varphi)$, where $u:=\cos \theta$, see e.g. [13, p. 85], we find that

$$
\begin{aligned}
& \frac{\partial}{\partial \eta}=\frac{e^{i \varphi}}{r \sqrt{2}}\left\{r \sqrt{1-u^{2}} \frac{\partial}{\partial r}+\frac{i}{\sqrt{1-u^{2}}} \frac{\partial}{\partial \varphi}-u \sqrt{1-u^{2}} \frac{\partial}{\partial u}\right\}, \\
& \frac{\partial}{\partial z}=u \frac{\partial}{\partial r}+\frac{1-u^{2}}{r} \frac{\partial}{\partial u} .
\end{aligned}
$$

Furthermore, we define the complex-valued unit vector

$$
\mathbf{e}_{\eta}:=\frac{1}{\sqrt{2}}\left(\mathbf{e}_{x}+i \mathbf{e}_{y}\right)
$$

and its complex conjugate counterpart $\mathbf{e}_{\eta}^{*}$, respectively, that obviously satisfy the following orthonormality relations:

$$
\begin{array}{lll}
\mathbf{e}_{\eta} \cdot \mathbf{e}_{\eta}^{*}=1, & \mathbf{e}_{\eta} \cdot \mathbf{e}_{\eta}=0, & \mathbf{e}_{\eta} \cdot \mathbf{e}_{z}=0 ; \\
\mathbf{e}_{\eta} \times \mathbf{e}_{\eta}^{*}=-i \mathbf{e}_{z}, & \mathbf{e}_{\eta} \times \mathbf{e}_{\eta}=\mathbf{0}, & \mathbf{e}_{\eta} \times \mathbf{e}_{z}=i \mathbf{e}_{\eta} .
\end{array}
$$

It is easily proved that the nabla operator can now be written in the form

$$
\boldsymbol{\nabla}=\mathbf{e}_{\eta}^{*} \frac{\partial}{\partial \eta}+\mathbf{e}_{\eta} \frac{\partial}{\partial \eta^{*}}+\mathbf{e}_{z} \frac{\partial}{\partial z}
$$

In the main part of this paper the treatment of the function $X_{l}^{m}(\mathbf{x})$, defined for $l, m \in \mathbb{Z}$ and $0 \leq l$ by

$$
\begin{array}{ll}
X_{l}^{m}(\mathbf{x}):=a_{l, m} r^{-l-1} Y_{l}^{m}(u, \varphi) & \text { for }|m| \leq l, \\
X_{l}^{m}(\mathbf{x}):=0 & \text { for }|m|>l
\end{array}
$$

with the spherical harmonics $Y_{l}^{m}(u, \varphi)$ from (103) and the constants

$$
a_{l, m}:=(-1)^{l+m} \sqrt{\frac{4 \pi}{2 l+1}(l+m) !(l-m) !},
$$

plays an important role. Its definition leads, together with the introduction of the coordinate $\eta$, to great simplifications of the calculations. Inserting (88) and (103) in (87) a more explicit expression of $X_{l}^{m}(\mathbf{x})$ for $|m| \leq l$ is given by

$$
X_{l}^{m}(\mathbf{x})=(-1)^{l}(l-m) ! r^{-l-1} P_{l}^{m}(u) e^{i m \varphi} .
$$


As an immediate consequence of the definitions in (87) and (88) we find that

$$
a_{l, m}=a_{l,-m}
$$

and, taking (106) into account, that

$$
X_{l}^{-m}(\mathbf{x})=(-1)^{m} X_{l}^{m^{*}}(\mathbf{x}) .
$$

Moreover, since $P_{0}^{0}(u)=1$, Eq. (89) implies that

$$
X_{0}^{0}(\mathbf{x})=\frac{1}{|\mathbf{x}|} .
$$

Next we derive properties of the derivatives of $X_{l}^{m}(\mathbf{x})$. Combinations of wellknown recurrence relations for the Legendre functions [12] lead to

$$
\begin{gathered}
\left(1-u^{2}\right) \frac{d P_{l}^{m}(u)}{d u}-(l+1) u P_{l}^{m}(u)=-(l+1-m) P_{l+1}^{m}(u), \\
u \sqrt{1-u^{2}} \frac{d P_{l}^{m}(u)}{d u}+(l+1) \sqrt{1-u^{2}} P_{l}^{m}(u)+\frac{m}{\sqrt{1-u^{2}}} P_{l}^{m}(u)=P_{l+1}^{m+1}(u) .
\end{gathered}
$$

(Note, that the definition of the Legendre functions $P_{l}^{m}(u)$ in [12] differs by a factor $(-1)^{m}$ from the one in [13] and this paper.) With these two equations, Eq. (83), and the expression of $X_{l}^{m}(\mathbf{x})$ in (89) we can show that for all $l, m \in \mathbb{Z}$ with $|m| \leq l$

$$
\begin{aligned}
\frac{\partial}{\partial z} X_{l}^{m}(\mathbf{x})= & (-1)^{l}(l-m) ! r^{-l-2} e^{i m \varphi}\left\{-(l+1) u+\left(1-u^{2}\right) \frac{\partial}{\partial u}\right\} P_{l}^{m}(u) \\
= & (-1)^{l+1}(l+1-m) ! r^{-l-2} e^{i m \varphi} P_{l+1}^{m}(u)=X_{l+1}^{m}(\mathbf{x}), \\
\frac{\partial}{\partial \eta} X_{l}^{m}(\mathbf{x})= & \frac{(-1)^{l}}{\sqrt{2}}(l-m) ! r^{-l-2} e^{i(m+1) \varphi} \\
& \times\left\{-(l+1) \sqrt{1-u^{2}}-\frac{m}{\sqrt{1-u^{2}}}-u \sqrt{1-u^{2}} \frac{\partial}{\partial u}\right\} P_{l}^{m}(u) \\
= & \frac{(-1)^{l+1}}{\sqrt{2}}(l-m) ! r^{-l-2} e^{i(m+1) \varphi} P_{l+1}^{m+1}(u)=\frac{1}{\sqrt{2}} X_{l+1}^{m+1}(\mathbf{x}),
\end{aligned}
$$

and finally with (91) and (95)

$$
\frac{\partial}{\partial \eta^{*}} X_{l}^{m}(\mathbf{x})=(-1)^{m}\left(\frac{\partial}{\partial \eta} X_{l}^{-m}(\mathbf{x})\right)^{*}=\frac{(-1)^{m}}{\sqrt{2}} X_{l+1}^{-m+1^{*}}(\mathbf{x})=-\frac{1}{\sqrt{2}} X_{l+1}^{m-1}(\mathbf{x}) .
$$

Obviously, the differential operators $\partial / \partial \eta, \partial / \partial \eta^{*}$, and $\partial / \partial z$ are ladder operators for $X_{l}^{m}(\mathbf{x})$. The fact that a differentiation of $X_{l}^{m}(\mathbf{x})$ means, in principle, nothing but a change of its order and degree, simplifies the treatment of this function considerably.

Another operator appearing in the main part of the text is: $\mathbf{x} \times \boldsymbol{\nabla}$. It can immediately be related to the well-known quantum mechanical angular momentum operator: $\mathbf{L}=\left(L_{x}, L_{y}, L_{z}\right)[13]$ by

$$
\mathbf{x} \times \boldsymbol{\nabla}=i \mathbf{L}=i\left\{\frac{\mathbf{e}_{\eta}^{*}}{\sqrt{2}} L_{+}+\frac{\mathbf{e}_{\eta}}{\sqrt{2}} L_{-}+\mathbf{e}_{z} L_{z}\right\},
$$


where for simplicity the Plank constant $\hbar$ has been dropped. Since $\mathbf{L}$ does not affect the spherical coordinate $r$, the application of the ladder operators $L_{ \pm}:=L_{x} \pm i L_{y}$ and $L_{z}$ on $X_{l}^{m}(\mathbf{x})$

$$
L_{z} X_{l}^{m}(\mathbf{x})=m X_{l}^{m}(\mathbf{x}), \quad L_{ \pm} X_{l}^{m}(\mathbf{x})=-(l \mp m) X_{l}^{m \pm 1}(\mathbf{x})
$$

immediately results from the well-known application on $Y_{l}^{m}(u, \varphi)[13$, p. 573].

Appendix C. Convergence problems. Here we prove that for $\hat{\mathbf{x}} \in \mathbb{R}^{3} \backslash C^{+}$, where $C^{+}$is defined in (51), the series representation

$$
\sum_{l=0}^{\infty} \sum_{m=-l}^{+l} \frac{4 \pi}{(2 l+1) a_{l, m}} r_{S}^{l} Y_{l}^{m^{*}}\left(u_{S}, \varphi_{S}\right) X_{l}^{m}(\hat{\mathbf{x}})
$$

of $\left|\hat{\mathbf{x}}-\mathbf{x}_{S}\right|^{-1}$ can be infinitely often differentiated term by term for $\hat{\eta}, \hat{\eta}^{*}$, and $\hat{z}$ (see Appendix B). By $\left(r_{S}, u_{S}, \varphi_{S}\right)$ and $(\hat{r}, \hat{u}, \hat{\varphi})$ with $u=\cos \theta$ we denote the spherical coordinates of $\mathbf{x}_{S}$ and $\hat{\mathbf{x}}$, respectively. It is sufficient to prove that the term-by-term differentiated series is again uniformly convergent for all $\mathbf{x}_{S} \in \partial \bar{C}$ and $\hat{\mathbf{x}} \in \mathbb{R}^{3} \backslash C^{+}$. Taking account of the definitions and results of $X_{l}^{m}(\mathbf{x})$ and $a_{l, m}$ stated in Appendix B and the estimation of $Y_{l}^{m}(u, \varphi)$ given in (105), we find for any $i_{1}, i_{2}, i_{3} \in\{0,1,2, \ldots\}$ that

$$
\begin{aligned}
& \left|\sum_{l=0}^{\infty} \sum_{m=-l}^{+l} \frac{4 \pi r_{S}^{l} Y_{l}^{m^{*}}\left(u_{S}, \varphi_{S}\right)}{(2 l+1) a_{l, m}}\left(\frac{\partial}{\partial \hat{\eta}}\right)^{i_{1}}\left(\frac{\partial}{\partial \hat{\eta}^{*}}\right)^{i_{2}}\left(\frac{\partial}{\partial \hat{z}}\right)^{i_{3}} X_{l}^{m}(\hat{\mathbf{x}})\right| \\
& \leq \frac{1}{2^{k_{3} / 2}} \sum_{l=0}^{\infty} \sum_{m=-l}^{+l} \frac{4 \pi\left|a_{l+k_{1}, m+k_{2}}\right|}{(2 l+1)\left|a_{l, m}\right|}\left|Y_{l}^{m^{*}}\left(u_{S}, \varphi_{S}\right)\right|\left|Y_{l+k_{1}}^{m+k_{2}}(\hat{u}, \hat{\varphi})\right| \frac{r_{S}^{l}}{\hat{r}^{l+k_{1}+1}} \\
& \leq \frac{1}{R_{C^{+}}^{k_{1}+1}} \sum_{l=0}^{\infty} \sum_{m=-l}^{+l} \prod_{k=1}^{k_{1}+k_{2}} \sqrt{l+m+k} \prod_{k=1}^{k_{1}-k_{2}} \sqrt{l-m+k}\left(\frac{r_{S}}{R_{C^{+}}}\right)^{l},
\end{aligned}
$$

where the abbreviations $k_{1}:=i_{1}+i_{2}+i_{3}, k_{2}:=i_{1}-i_{2}$, and $k_{3}:=i_{1}+i_{2}$ have been used. With

$$
\prod_{k=1}^{k_{1}+k_{2}} \sqrt{l+m+k} \prod_{k=1}^{k_{1}-k_{2}} \sqrt{l-m+k} \leq \prod_{k=1}^{2 k_{1}} \sqrt{(l+k)^{2}-m^{2}} \leq\left(l+2 k_{1}\right)^{2 k_{1}}
$$

the continuation of the estimation in (100) finally results in the series

$$
\leq \frac{1}{R_{C^{+}}^{k_{1}+1}} \sum_{l=0}^{\infty}(2 l+1)\left(l+2 k_{1}\right)^{2 k_{1}}\left(\frac{r_{S}}{R_{C^{+}}}\right)^{l}
$$

that converges absolutely and independently of $\mathbf{x}_{s}$ and $\hat{\mathbf{x}}$ and for all $k_{1} \in\{0,1$, $2, \ldots\}$ because $r_{S}<R_{C^{+}}$.

Appendix D. Important properties of spherical harmonics. For $l, m \in \mathbb{Z}$ and $0 \leq l$ the spherical harmonics are defined by [13]

$$
\begin{array}{ll}
Y_{l}^{m}(u, \varphi):=(-1)^{m} \sqrt{\frac{(2 l+1)}{4 \pi} \frac{(l-m) !}{(l+m) !}} P_{l}^{m}(u) e^{i m \varphi} & \text { for }|m| \leq l, \\
Y_{l}^{m}(u, \varphi):=0 & \text { for }|m|>l,
\end{array}
$$


where $P_{l}^{m}(u)$ denotes the associated Legendre functions. Note that the factor $(-1)^{m}$ is sometimes, e.g. in [12], incorporated in the definition of $P_{l}^{m}(u)$. The spherical harmonics satisfy the orthogonality relation

$$
\int_{-1}^{+1} \int_{0}^{2 \pi} Y_{l^{\prime}}^{m^{\prime}}(u, \varphi) Y_{l}^{m^{*}}(u, \varphi) d \varphi d u=\delta_{l, l^{\prime}} \delta_{m, m^{\prime}}
$$

Based on a result for Legendre functions [14] an upper bound for the spherical harmonics reads

$$
\left|Y_{l}^{m}(u, \varphi)\right| \leq \sqrt{\frac{2 l+1}{4 \pi}} .
$$

The relation between $P_{l}^{m}$ and $P_{l}^{-m}[13]$ implies that

$$
Y_{l}^{-m}(u, \varphi)=(-1)^{m} Y_{l}^{m^{*}}(u, \varphi) .
$$

The generating function of the spherical harmonics [13]

$$
\frac{1}{\left|\mathbf{x}-\mathbf{x}^{\prime}\right|}=\sum_{l=0}^{\infty} \sum_{m=-l}^{+l} \frac{4 \pi}{2 l+1} Y_{l}^{m^{*}}(u, \varphi) Y_{l}^{m}\left(u^{\prime}, \varphi^{\prime}\right) \cdot \begin{cases}\frac{\left(r^{\prime}\right)^{l}}{r^{l+1}} & \text { for } r>r^{\prime}, \\ \frac{r^{l}}{\left(r^{\prime}\right)^{l+1}} & \text { for } r<r^{\prime}\end{cases}
$$

converges uniformly for fixed $r$ and $r^{\prime}$, if $r \neq r^{\prime}$.

\section{REFERENCES}

[1] D. M. Herlach, G. Lohöfer, R. Knauf, J. Piller, and P. Preu, Electromagnetic positioning and inductive heating under micro-g, Proceedings of the 6th European Symposium on Material Sciences under Microgravity Conditions, Bordeaux, France, December 1986, European Space Agency SP-256, 1987, pp. 437-443

[2] G. Lohöfer, P. Neuhaus, and I. Egry, TEMPUS-A facility for measuring thermophysical properties of undercooled liquid metals, to appear in: High Temp.-High Press. 23 (1991)

[3] I. Egry, B. Feuerbacher, G. Lohöfer, and P. Neuhaus, Viscosity measurement in undercooled metallic melts, Proceedings of the VIIth European Symposium on Materials and Fluid Sciences in Microgravity, Oxford, UK, September 1989, European Space Agency SP-295, 1990, pp. 257-260

[4] G. Lohöfer, Device for positioning and melting electrically conductive materials without a receptacle, US Patent No. 4979182, 1990

[5] F. R. Block and A. Theissen, Das elektromagnetische Schwebeschmelzen-ein Beitrag zu den Verfahren des tiegelfreien Schmelzens, Elektrowärme International 29, 349-354 (1971)

[6] W. Brisley and B. S. Thornton, Electromagnetic levitation calculations for axially symmetric systems, British J. Appl. Phys. 14, 682-686 (1963)

[7] E. Fromm and $\mathrm{H}$. Jehn, Electromagnetic forces and power absorption in levitation melting, British J. Appl. Phys. 16, 653-663 (1965)

[8] P. R. Rony, The electromagnetic levitation of metals, Trans. Vacuum Met. Conference 1964 (ed. by M. A. Cocca), Amer. Vacuum Society, Boston, MA, 1965, pp. 55-135

[9] G. Lohöfer, Theory of an electromagnetically levitated metal sphere: Absorbed power, SIAM J. Appl. Math. 49, 567-581 (1989)

[10] J. D. Jackson, Classical electrodynamics, Wiley, New York, 1975

[11] R. Courant and D. Hilbert, Methoden der mathematischen Physik, vol. 2, Springer, Berlin, 1968

[12] M. Abramowitz and I. A. Stegun, Handbook of mathematical functions, Dover, New York, 1970

[13] G. Arfken, Mathematical methods for physicists, Academic Press, New York, 1970

[14] G. Lohöfer, Inequalities for Legendre functions and Gegenbauer functions, J. Approx. Theory 64, 226-234 (1991) 